\title{
A search for new hot subdwarf stars by means of Virtual Observatory tools
}

\author{
R. Oreiro ${ }^{1}$, C. Rodríguez-López ${ }^{2,3}$, E. Solano ${ }^{4}$, A. Ulla ${ }^{3}$, R. Østensen ${ }^{5}$, and M. García-Torres ${ }^{6}$
}

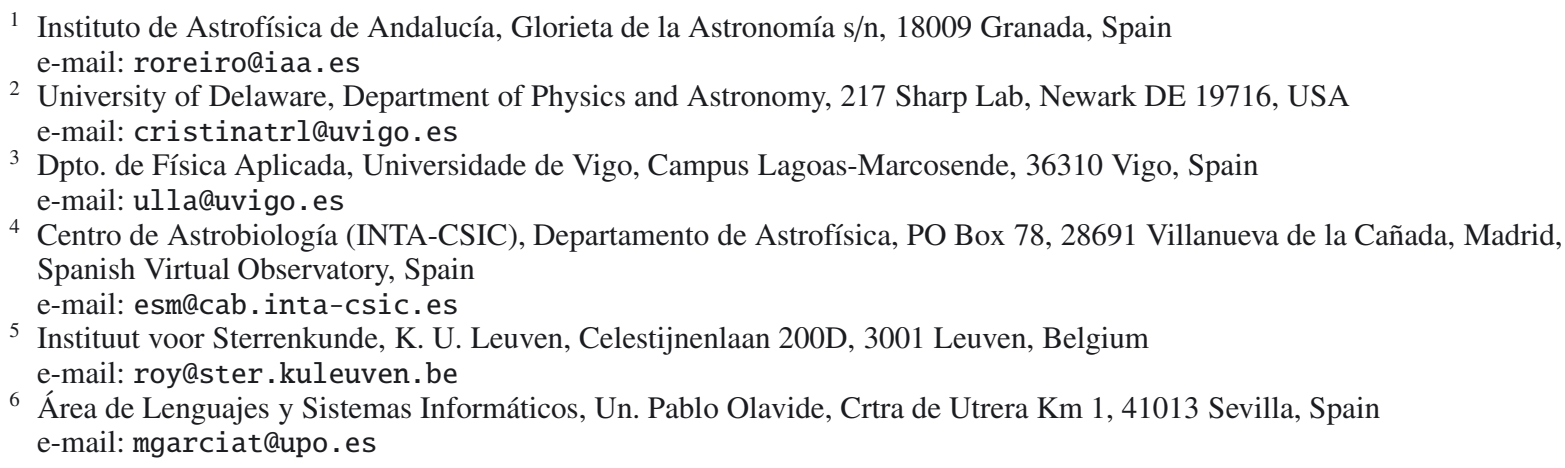

Received 14 December 2010 / Accepted 8 March 2011

\begin{abstract}
Context. Recent massive sky surveys in different bandwidths are providing new opportunities to modern astronomy. The Virtual Observatory (VO) provides the adequate framework to handle the huge amount of information available and filter out data according to specific requirements.

Aims. Hot subdwarf stars are faint, blue objects, and are the main contributors to the far-UV excess observed in elliptical galaxies. They offer an excellent laboratory to study close and wide binary systems, and to scrutinize their interiors through asteroseismology, since some of them undergo stellar oscillations. However, their origins are still uncertain, and increasing the number of detections is crucial to undertake statistical studies. In this work, we aim at defining a strategy to find new, uncatalogued hot subdwarfs.

Methods. Making use of VO tools we thoroughly search stellar catalogues to retrieve multi-colour photometry and astrometric information of a known sample of blue objects, including hot subdwarfs, white dwarfs, cataclysmic variables and main-sequence OB stars. We define a procedure to distinguish among these spectral classes, which is particularly designed to obtain a hot subdwarf sample with a low contamination factor. To check the validity of the method, this procedure is then applied to two test sky regions: to the Kepler FoV and to a test region of $300 \mathrm{deg}^{2}$ around $(\alpha: 225, \delta: 5)$ deg.

Results. As a result of the procedure we obtained 38 hot subdwarf candidates, 23 of which had already a spectral classification. We have acquired spectroscopy for three other targets, and four additional ones have an available SDSS spectrum, which we used to determine their spectral type. A temperature estimate is provided for the candidates based on their spectral energy distribution, considering two-atmospheres fit for objects with clear infrared excess as a signature of the presence of a cool companion. Eventually, out of 30 candidates with spectral classification, 26 objects were confirmed to be hot subdwarfs, which means a contamination factor of only $13 \%$. The high rate of success demonstrates the validity of the proposed strategy to find new uncatalogued hot subdwarfs. An application of this method to the entire sky will be presented in a forthcoming work.
\end{abstract}

Key words. stars: early-type - subdwarfs - astronomical databases: miscellaneous - Virtual Observatory tools

\section{Introduction}

Hot subdwarf stars (hot sds), objects with temperatures exceeding $19000 \mathrm{~K}$ and $\log g \geq 5$, are considered to be the field counterparts of the extended horizontal-brach (EHB) stars found in globular clusters. This evolutionary state implies a $\sim 0.5 M_{\odot}$ canonical mass and a core He-burning structure with a very thin H-envelope $\left(M_{\mathrm{env}} \leq 0.02 M_{\odot}\right.$, Heber 1986). This structure prevents the hot sds from ascending the asymptotic giant branch (AGB) and, once the core helium is exhausted, they evolve towards hotter temperatures before they reach degeneracy and cool as a normal white dwarf star (Dorman et al. 1993).

Whereas the role of hot subdwarfs as white dwarf progenitors is well understood, the circumstances that lead to the removal of all but a tiny fraction of the hydrogen envelope, at about the same time as the core has achieved the mass required for the He flash $\left(\approx 0.47 M_{\odot}\right)$, are still a matter of debate. Two main scenarios have been proposed to explain the formation of these objects: i) enhancement of the mass loss efficiency near the red giant branch (RGB) tip (D'Cruz et al. 1996); ii) mass transfer through binary interaction (Mengel et al. 1976).

A large percentage of hot subdwarfs are observationally found in binary systems (Ulla \& Thejll 1998; Maxted et al. 2001), which supports the mass transfer scenario. However, a non negligible percentage appear as single hot sds. From a single evolution point of view, it is unknown, though, what could cause an enhanced mass loss at the RGB. The binary population synthesis by Han et al. $(2002,2003)$ shows that common envelope ejection (CEE), stable Roche lobe overflow and helium white dwarfs merging can produce hot sds in close or wide 
binaries, as well as single sds. These different paths produce distinct orbital period distributions and a variety of mass ranges for the hot sds and binary companions. A confrontation of theory and observations could be the key to clarify the hot sds evolutionary state and, moreover, help to fine-tune the processes related to the CEE. Some attempts have already been made in this direction (Morales-Rueda et al. 2003; Stroeer et al. 2007), but the observed orbital period distribution is biased towards the close binary systems (with higher radial velocity variations), and the mass distribution is far from being statistically significant. Besides, the distribution of the companion spectral type also suffers from biases associated to the catalogue from which they were selected (see e.g. Wade et al. 2009).

The total mass of some hot sds could be inferred for the few eclipsing binary systems known (Østensen et al. 2010a; For et al. 2010). Thanks to the existence of stellar pulsations in some Btype hot sds ( $\mathrm{sdBs}$ ), the total mass could also be determined using asteroseismic tools for a handful of cases (Randall et al. 2009 , and references therein). Unfortunately, there are few shortperiod sdB pulsators with a sufficient number of excited modes to perform an asteroseismic analysis. However, this may be overcome soon, since we are entering the age of space based asteroseismology. Missions like CoRoT (Auvergne et al. 2009) and Kepler (Borucki et al. 2010) have eventually opened the door to asteroseismology of the long-period sdB pulsators (Charpinet et al. 2010; Van Grootel et al. 2010), which are more numerous than short-period ones, but far more challenging for groundbased observations.

The exciting discovery of the first O-type pulsating hot sd (sdO, Woudt et al. 2006) has been somehow tarnished by the fact that no other similar objects have been found up to now. In spite of the extensive searches that have been performed (RodríguezLópez et al. 2007), new discoveries are hindered by the lack of catalogued sdOs in the temperature range of the unique pulsator, $T_{\text {eff }} \sim 70000 \mathrm{~K}$ (Fontaine et al. 2008; Rodríguez-López et al. 2010). O-type hot subdwarfs are less numerous in general than sdBs and, in particular, only about 40 catalogued sdOs have a temperature estimate within $\mathrm{a} \pm 5000 \mathrm{~K}$ box around the unique sdO pulsator. Much fewer match a similar $\log g$ and helium abundance.

The scarcity of catalogued sdOs at this temperature range may in part be attributed to the historical difficulty in obtaining NLTE model atmosphere grids with $T_{\text {eff }}$ values over $60000 \mathrm{~K}$, firstly overcome by Dreizler et al. (1990). Since then, only a few quantitatively significant spectral analysis of sdOs were undertaken (Thejll et al. 1994; Bauer \& Husfeld 1995; Stroeer et al. 2007). The deficit in pulsating sdOs may be owing to sdOs having different evolutionary channels and/or large chemical inhomogeneities. Stroeer et al. (2007) found that sdOs with subsolar $\mathrm{He}$ abundances never showed $\mathrm{C}$ or $\mathrm{N}$ lines and were scattered in the HR diagram. On the other hand, sdOs with supersolar He abundances always showed $\mathrm{C}$ and/or $\mathrm{N}$ lines and similar parameters around $T_{\text {eff }} \sim 50000 \mathrm{~K}$ and $\log g \sim 5.5$.

Increasing the number ratio of hot sds in the different galactic populations may help to sort out their origins, as suggested by Altmann et al. (2004): the binary scenario of Mengel et al. (1976) would be favoured if the ratio of sdBs in the halo, thin and thick disk is similar to that of other evolved stars; on the contrary, if the extensive mass loss scenario proposed by D'Cruz et al. (1996) is dominant, sdBs in the disk should be more numerous compared to other mid-temperature HB stars. Some light could be shed on the formation processes if the low number of known halo and thin disk sdBs could be rised. Note that, as described in
Sect. 2, most surveys for faint blue targets intentionally avoided the galactic disk to diminish contamination with OB stars.

The aim of this work is to devise a procedure to identify new hot sds with the lowest contamination factor. Our main ally will be the Virtual Observatory ${ }^{1}$ (VO), which is becoming an essential, thourough, and time-saving tool in aid of the overwhelmed-by-data astronomers. Modern observational astronomy profit from large area, multiwavelength surveys, whose data are stored in different archives and formats. Although data can be queried through different access methods, the lack of interoperability among astronomical services can hinder one from profiting the most from of combined information from several surveys. These drawbacks can be overcome if we work in the framework of the Virtual Observatory, an international initiative designed to provide the astronomical community with the data access and the research tools necessary to enable the exploration of the digital, multi-wavelength universe that is resident in the astronomical data archives.

We make use of VO tools throughout the paper to benefit from an easy data access and analysis for our scientific purpose. We overview the conventional catalogues used to select hot subdwarfs in Sect. 2. In Sect. 3 we describe our devised method to search for hot sds. In Sect. 4 we present an application of the method to the Kepler FoV and a test region as well as the results obtained from the spectroscopic follow-up of our list of candidates. In Sect. 5 we pay attention to binary hot sds candidates. Finally, in Sect. 6 we summarize our findings.

\section{Hot subdwarf surveys}

Surveys in search for faint blue stars began and flourished around the 60's. Greenstein (1960) gathered under the term faint blue stars all not-well understood spectra of stars that sat below the main sequence in the HR diagram. Intense surveys for new blue subluminous stars followed the pioneer discoveries of Humason \& Zwicky (1947), among them, Feige (1958) searched for faint blue stars brighter that $B_{\mathrm{pg}}=14 \mathrm{mag}$, within $6000 \mathrm{deg}^{2}$ around both galactic poles and found 114 objects, which were later spectroscopically analysed by Sargent \& Searle (1968). Haro \& Luyten (1962) published the Faint Blue Stars near the South Galactic Pole, a catalogue with about 8700 stars, based on the Johnson photometric indices, up to magnitude 19, comprising hot sds, white dwarfs (WDs) and quasars, and for which photometric indices, spectroscopic and proper motion data were given. Greenstein (1966) performed a spectroscopic study of about a hundred faint blue stars mainly at the galactic poles from the catalogues of Humason \& Zwicky (1947), Iriarte \& Chavira (1957), Chavira (1958) and Feige (1958), which allowed for the first time a distinction between hot sds, WDs, and halo or horizontal branch stars. The most recent catalogues of hot sds used nowadays are described below.

\subsection{The Palomar-Green (PG) catalogue of ultraviolet-excess stellar objects}

The Green et al. (1986) $U-B$ photographic survey lists about 1900 objects with a limiting magnitude $B_{\mathrm{pg}}=16.7$ mag covering about $11000 \mathrm{deg}^{2}$ at Galactic latitudes $|b|>30^{\circ}$ and declinations $\delta<-10^{\circ}$. A total of 1715 objects showing ultraviolet excess, given by $(U-B)_{\mathrm{pg}}<-0.46$ were observed spectroscopically for classification. This yielded over 900 hot sds, which make up $\sim 53 \%$ of the catalogue objects.

1 http://www.ivoa.net 


\subsection{The Kitt Peak-Downes (KPD) survey for galactic plane ultraviolet-excess objects}

Downes (1986) found 60 hot sds ( 40\% of the objects) and 10 WDs from a $1000 \mathrm{deg}^{2}$ two-colour photographic and spectroscopic survey of the Galactic plane, obtaining spectra for about 700 UV-excess candidates. Accurate space densities could be determined for the first time for Galactic plane UV-excess objects (i.e. hot sds, WDs and cataclysmic variables), because of the homogeneity of the sample, which was complete to $B_{\mathrm{pg}}=$ $15.3 \mathrm{mag}$.

\subsection{The Montreal-Cambridge-Tololo (MCT) survey of southern subluminous blue stars}

The Montreal-Cambridge-Tololo photographic and spectroscopic Survey of southern subluminous blue stars (Lamontagne et al. 2000; Demers et al. 1986) covers $\sim 6800 \mathrm{deg}^{2}$ centred on the south Galactic polar cap, at latitudes below $b=-30^{\circ}$ not covered by the PG survey, and is complete down to $B_{\mathrm{pg}}=$ $16.5 \mathrm{mag}$. The criterion for selecting candidates was $(U-B)_{\mathrm{pg}} \leq$ -0.6 , which leads to some 3000 objects, for a third of which spectroscopy was performed. Results for the analysis of the region of $\sim 800 \mathrm{deg}^{2}$ of the south Galactic cap are given: of $188 \mathrm{ob}-$ jects, $40 \%$ were found to be hot sds.

\subsection{A catalogue of spectroscopically identified hot subdwarfs}

Kilkenny et al. (1988) made the considerable effort of collecting 1225 known hot sds spectroscopically identified from different literature sources and, in that moment, yet to be published data. The main sources for this compilation are the PG and KPD surveys. This was the most extensive hot sds catalogue until the release of the Subdwarf Database (Østensen 2006, see below).

\subsection{The Hamburg-Schmidt/ESO (HQS/HES) quasar survey}

The Hamburg-Schmidt Survey (Hagen et al. 1995), with the prime scientific goal of providing new, bright QSOs, has also been a source of new hot sds. The prime scientific goal of the northern survey $\left(\sim 14000 \mathrm{deg}^{2}\right)$ is to provide a complete sample of bright, high-redshift QSOs, to expand the PG-survey in area and depth up to $B<17 \mathrm{mag}$. The quasar search was extended to the southern sky (the Hamburg/ESO Survey, Wisotzki et al. 1991; Wisotzki 1994; Wisotzki et al. 2000), where it aims at covering $\sim 5000 \mathrm{deg}^{2}$ for sources with $B<16.5 \mathrm{mag}$. In addition, the digitized data base is currently used in the search for hot stars by the Hamburg-Bamberg-Kiel collaboration (see e.g. Heber et al. 1991; Edelmann et al. 2003). Candidate hot stars, for which a follow-up and later analyses were performed, were selected on the basis of bluest spectra and visual classification. A initial candidate list of 400 objects yielded $~ 50 \%$ hot sds (Edelmann et al. 2003).

\subsection{Edinburgh-Cape (EC) survey}

The Edinburgh-Cape Survey (Stobie et al. 1997; Kilkenny et al. 1997, 2010) aim is to discover blue stellar objects brighter than $B \sim 18$ in southern sky Galactic latitudes $|b|>30^{\circ}$ and declination $\delta<-12.5$, meaning $\sim 8000 \mathrm{deg}^{2}$.

The criterion to select blue stellar objects from UK Schmidt telescope plates is $(U-B)_{\mathrm{pg}}<-0.4$. The survey was divided into six zones, each comprising $\sim 1500 \mathrm{deg}^{2}$. The first release of the survey (Kilkenny et al. 1997) gives results for the analysis of Zone 1, yielding 675 hot blue objects with a $45 \%$ of hot sds. For the most up-to-date status of the project, we refer the reader to Kilkenny et al. (2010)

\subsection{SPY - the ESO supernova type la progenitor survey}

The SPY (Napiwotzki et al. 2003) is a survey designed to search for short-period binary WDs as potential progenitors of type Ia supernovae. The SPY obtained accurate radial velocities for WD candidates brighter than $B=16.5$ mag that belong to a variety of source catalogues, mainly the McCook \& Sion (1999), but also the HQS/HES, MCT, and EC catalogues. Napiwotzki et al. (2004) found $46 \mathrm{sdBs}$ and $23 \mathrm{sdOs}$ to be owing to misclassifications in the input catalogs.

\subsection{The subdwarf database}

The Subdwarf Database ${ }^{2}$ (Østensen 2006) is the latest compilation of any object ever classified as a hot subdwarf. Initially based on the compendium by Kilkenny et al. (1988), it is in a continuous process of up-dating, and today contains more than 2400 entries. For each entry, the database provides links to finding charts, the SIMBAD astronomical database, and data available in the literature, namely $T_{\text {eff }}$, surface gravity, helium abundance, photometry, and spectral classification. A quality flag is given for the derived spectral classes, to give an estimate of the reliability of the determination.

Given that the Subdwarf Database is the most complete compilation of hot sds, it is of invaluable help in any study aiming at detecting any yet unclassified hot subdwarf, as we are attempting here.

\section{The search method}

The main objective of this work is to design a procedure to identify new hot subdwarfs. Special care is taken to avoid contamination from other types of objects by giving more importance to the successful rate (low contamination factor) than to the completeness of the sample of new hot sds found. Former faint blue star catalogues that were used to select hot sds also had a high percentage of WDs. To name but a few: the PG catalogue had $\sim 50 \%$ of hot sds, but featured a $25 \%$ of WDs; the MCT survey showed $\sim 40 \%$ of hot sds and $\sim 30 \%$ of WDs; whereas the EC survey of Zone 1 showed $45 \%$ of hot sds and 15\% of WDs.

Because we aim at obtaining a subdwarf candidate sample as pure as possible, we define the best strategy using spectroscopically classified bona fide catalogues:

- the Subdwarf Database (Østensen 2006) as the hot sds sample;

- SDSS4 confirmed White Dwarf catalogue (Eisenstein et al. 2006) to obtain a list of white dwarfs;

- the Catalogue of Cataclysmic Variables (Downes et al. 2006);

- the Photometry and spectroscopy for luminous stars catalogue (Reed 2005) to obtain main-sequence OB stars.

We considered WDs, cataclysmic variables (CVs), and OB stars because they have a photometric signature similar to that of hot subdwarfs and represent, therefore, the main sources of pollution

2 http://www.ing.iac.es/ds/sddb/ 
Table 1. Number of initial hot sds, WDs, CVs, and OB targets used for defining the procedure in Sect. 3, including the number of objects with available data in GALEX, 2mAss and SuperCOSMOS.

\begin{tabular}{lcccc}
\hline \hline & Hot sds & WDs & CVs & OBs \\
\hline Initial nums. in cats. & 2430 & 9277 & 1578 & 9123 \\
2MASs & $1985(82 \%)$ & $680(7 \%)$ & $956(61 \%)$ & $7504(82 \%)$ \\
GALEX & $1578(65 \%)$ & $5798(62 \%)$ & $460(29 \%)$ & $42(0.46 \%)$ \\
SuperCOSMOS & $2243(92 \%)$ & $8636(93 \%)$ & $1145(73 \%)$ & $5049(55 \%)$ \\
2MASS+GALEX & $1246(51 \%)$ & $445(5 \%)$ & $292(18 \%)$ & $40(0.44 \%)$ \\
2MASS+GALEX+SuperC. & $1162(48 \%)$ & $421(4 \%)$ & $274(17 \%)$ & $33(0.36 \%)$ \\
\hline
\end{tabular}

in our study. Table 1 indicates the number of targets in each input catalogue.

The methodology proposed makes use of existing data from different surveys. The data gathering is described in Sect. 3.1, which is then used to filter out non hot sds: combined 2mass and GALEX photometry (Sect. 3.2) will reject red targets and a large percentage of contaminators; proper motion information (Sect. 3.3) will help to distinguish between kinematic populations, and eventually a temperature estimate given by the fit to the spectral energy distribution (Sect. 3.4) will further improve the selection of hot sds.

\subsection{Archive data gathering}

We made use of TOPCAT ${ }^{3}$, a VO-tool to work with tabular data, to access and download the GALEX ${ }^{4}$ and 2 MAss $^{5}$ (Morrissey et al. 2007; Skrutskie et al. 2006) photometric magnitudes for all sources in the input catalogues. Only the best coordinate match within 5 arcsec was considered.

We selected only those targets with both 2MAss and GALEX photometry, which significantly limited the test sample. Only a low fraction of the sample WDs has 2mass photometry because they are generally too faint at these wavelengths, while for OB stars, there is GALEX photometry for very few targets because GaLEX does not cover the galactic plane. Table 1 lists the number of targets with available photometric magnitudes for every input list (see also Fig. 1) and those with photometry in the two surveys (under 2MAss+GALEX).

The GALEX and 2mass apparent magnitudes ( $F U V$ and $N U V$, and $K \mathrm{~s}$, respectively) were corrected for galactic extinction using the $E(B-V)$ values from Schlegel et al. (1998) and applying the corresponding correction factors by Wyder et al. (2005) for $F U V$ and $N U V$ GaLEX filters, and Cardelli et al. (1989) for the 2mass $K$ s filter:

$$
\begin{aligned}
& F U V_{0}=F U V-A_{F U V}=F U V-8.376 E(B-V) \\
& N U V_{0}=N U V-A_{N U V}=N U V-8.741 E(B-V) \\
& K \mathrm{~s}_{0}=K \mathrm{~s}-A_{K \mathrm{~s}}=K \mathrm{~s}-0.114 E(B-V),
\end{aligned}
$$

where the 0 subscripts indicate extinction-corrected magnitudes.

Moreover, we also downloaded the SuperCOSMOS ${ }^{6}$ (Hambly et al. 2001) proper motions for the input list of targets, which will be used to separate different kinematic populations. A high percentage of the test objects have catalogued proper motions, as seen in Table 1. From now on, we will only use targets that have 2mass and GALEx photometry and proper motions given by SuperCOSMOS (under 2MASS+GALEX+SuperC in Table 1). This leaves us with a low fraction of WDs and a tiny

\footnotetext{
${ }^{3}$ http://www.star.bris.ac.uk/ mbt/topcat/

4 http://galex.stsci.edu/GR4/

5 http://www.ipac. caltech.edu/2mass/

${ }^{6}$ http://surveys.roe.ac.uk/ssa/
}

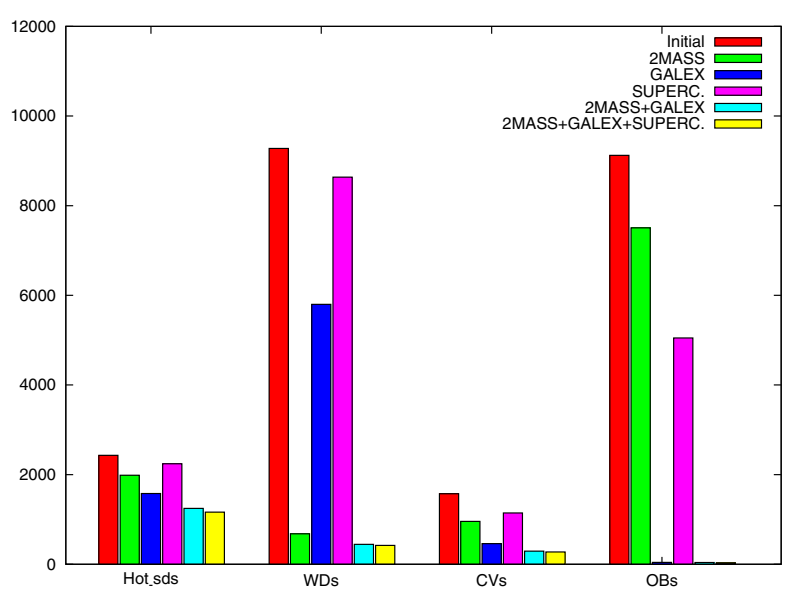

Fig. 1. Histogram showing the initial number of objects in the input catalogues, those with a counterpart in the databases used, and subsequent remaining numbers as cross-correlation between catalogues are done. Numbers are taken from Table 1.

fraction of OBs with respect to the original input catalogues, but we also lose about half of the initial hot sds.

\subsection{Ultraviolet-infrared colour filter}

Rhee et al. (2006) already presented a selection strategy to identify hot subdwarfs using a combination of photometric indices from GALEX and 2mass. The GALEX satellite is performing a series of sky surveys in two ultraviolet bands, $F U V$ (1344-1786 $\AA$ ) and $N U V(1771-2831 \AA)$, whereas 2MAss scanned the entire sky in three near-infrared bands, $J(1.25 \mu \mathrm{m})$, $H(1.65 \mu \mathrm{m})$, and $K \mathrm{~s}(2.17 \mu \mathrm{m})$. Given their large area coverage, the combination of both datasets represents an excellent approach to separate blue from red targets.

Rhee et al. (2006) cross-matched the GALEx and 2MAss catalogues in a $3500 \mathrm{deg}^{2}$ region. They proposed as hot sds candidates those falling within the limits $-4<(F U V-K s)<1$ and $-1.5<(F U V-N U V)<0.5$ in a two-colour diagram. However, follow-up spectroscopic observations for a subsample of 34 subdwarf candidates resulted in $60 \%$ contamination from other blue objects. We will therefore attempt to use slightly refined search criteria.

Following Rhee et al. (2006), we plotted $\left(F U V_{0}-K s_{0}\right)$ vs. $\left(F U V_{0}-N U V_{0}\right)$ for the input sample (Fig. 2). The four different object classes under study are plotted with different colours and symbols. The black dashed box indicates the limits for the hot sds selection proposed by Rhee et al. (2006). It results in a very effective procedure to differentiate hot sds from the other samples: $92 \%$ of the considered hot sds lie within this box, while only $12 \%$ of the WDs, $24 \%$ of the CVs and $45 \%$ of the OBs fulfil this selection criterion. 
R. Oreiro et al.: A search for new hot subdwarf stars by means of Virtual Observatory tools

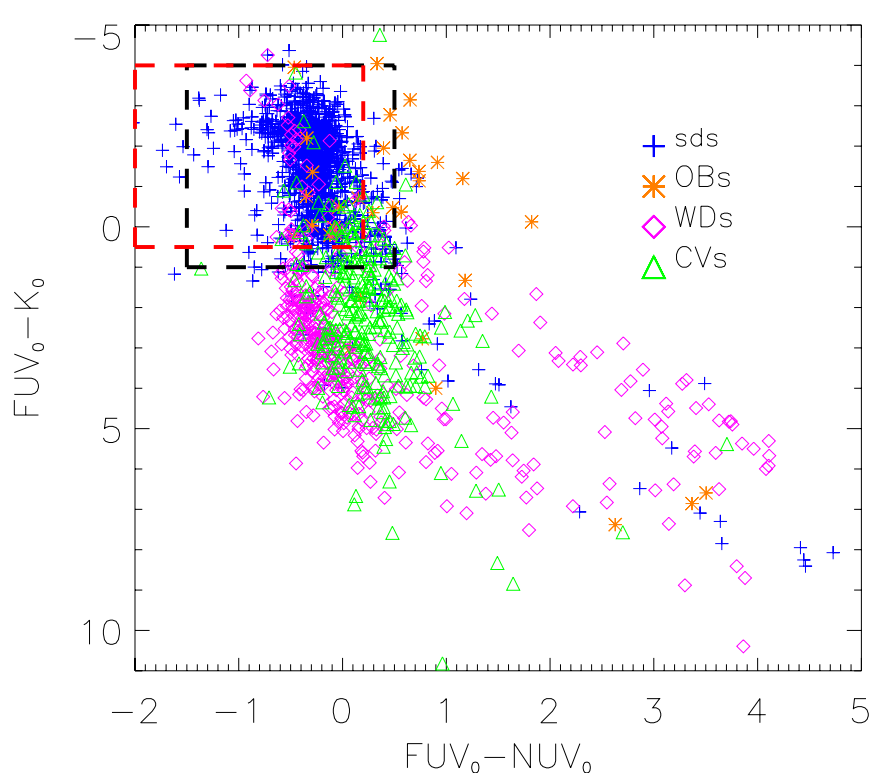

Fig. 2. Test sample of hot sds, WDs, CVs, and OBs having 2MASS+GALEX+SuperCOSMOS data. Note that those hot sds with $F U V_{0}-N U V_{0}>2$ all have a $F U V$ magnitude above 18.7, close to or above the limiting magnitude, which is causing their red appearance. The black dashed box comprises objects within the GALEX-2MASs selection limits given by Rhee et al. (2006). The red dashed box gives selection limits proposed in this work. See text and Table 2 for further details.

Slight changes on the box limits result in a purer or more contaminated hot sds sample, always at the expense of sacrifying good candidates. After some tests, we redefined Rhee's selection box as

$$
\begin{aligned}
& -4<\left(F U V_{0}-K s_{0}\right)<0.5 \\
& -2<\left(F U V_{0}-N U V_{0}\right)<0.2,
\end{aligned}
$$

which is a good compromise between obtaining a low contamination factor and avoiding the rejection of too many hot sds. The new defined selection limits are overploted as a red dashed box in Fig. 2. Table 2 compares the percentage of targets surviving the two different selection criteria. As indicated, the new limits diminish contamination, in particular from CVs and OBs.

Given that white dwarfs can be nearby objects, their magnitudes might have been overcorrected for galactic extinction. As a cross-check, if half of the $E(B-V)$ values from Schlegel et al. (1998) were used, the resulting $F U V_{0}-K_{0}$ colours would be shifted towards blue $\sim 0.15$ mag on average, and only one new white dwarf would enter the selection criteria indicated in Fig. 2.

\subsection{Proper-motion filter}

Further improvement to this colour-colour diagram is obtained when reduced proper motions (RPM) are used to separate populations of stars with different kinematics. The RPM in any filter is calculated as

$H($ Filter $)=m+5 \log _{10}(\mu)+5$

where $m$ is the apparent magnitude for the corresponding filter and $\mu$ the proper motion measured in milliarcsec per year. Salim \& Gould (2002) used a combination of optical and infrared RPMs to achieve a fairly good isolation for the bluest
Table 2. Percentage of input objects fulfilling the selection criteria.

\begin{tabular}{lcccc}
\hline \hline & Hot sds & WDs & CVs & OBs \\
\hline${ }^{1}$ Rhee et al. criteria & & & & \\
$-4<\left(F U V_{0}-K s_{0}\right)<1$ & $92 \%$ & $12 \%$ & $24 \%$ & $45 \%$ \\
$-1.5<\left(F U V_{0}-N U V_{0}\right)<0.5$ & & & & \\
${ }^{2}$ This paper & & & & \\
$-4<\left(F U V_{0}-K s_{0}\right)<0.5$ & $87 \%$ & $10 \%$ & $13 \%$ & $33 \%$ \\
$-2<\left(F U V_{0}-N U V_{0}\right)<0.2$ & & & & \\
${ }^{3}$ This paper & & & & \\
$19<H\left(N U V_{0}\right)<27$ & $83 \%$ & $3 \%$ & $11 \%$ & $21 \%$ \\
${ }^{4}$ Total number after $2 \& 3$ & 960 & 14 & 30 & 7 \\
${ }^{5}$ VOSA $T_{\text {eff }}>19000^{a}$ & $64(72) \%$ & $3(3) \%$ & $1(4) \%$ & $3(6) \%$ \\
${ }^{6}$ Total number after 2, 3 \& $5^{a}$ & $749(846)$ & $14(14)$ & $3(12)$ & $1(2)$ \\
\hline
\end{tabular}

Notes. Percentages refer to objects with data in 2MASS+GALEX+SuperCOSMOS catalogues (see Table 1 for further details). ${ }^{(1)}$ Percetage of input objects fulfilling the two-colour indices $\left(F U V_{0}-K \mathrm{~s}_{0}\right)-\left(F U V_{0}-N U V_{0}\right)$ selection criterion given by Rhee et al. (2006). (2) Same as in ${ }^{(1)}$ but for the criterion proposed in this paper. ${ }^{(3)}$ Reduced proper motion filter. ${ }^{(4)}$ Same as in ${ }^{(3)}$ but in absolut numbers. ${ }^{(5)}$ Objects after the temperature filter. ${ }^{(6)}$ Same as in ${ }^{(5)}$ but in absolut numbers. ${ }^{(a)}$ In parenthesis: considering also objects with a bad SED fit (see Sect. 3.4).

WDs from main-sequence stars and (cool) subdwarfs, although contamination factors were not given.

With this aim, we constructed an UV-RPM diagram for the test catalogues. In the upper panel of Fig. 3 we plot $H\left(N U V_{0}\right)$ against $\left(F U V_{0}-N U V_{0}\right)$ for the complete sample. Subdwarfs can be distinguished from OB main-sequence stars because they are several magnitudes dimmer at the same colour and typically have higher velocities. These effects tend to move hot sds significantly below the OB stars in the reduced proper-motion diagram. White dwarfs, with even fainter magnitudes, also appear clearly separated. Cataclysmic variables, on the other hand, quite overlap with hot sds and WDs.

In the lower panel of Fig. 3 only targets fulfilling Eqs. (4), (5) (see Sect. 3.2) are included. We now select targets within $19<H\left(N U V_{0}\right)<27$, to end up with a sample almost devoid of WDs, containing only a $3 \%$ of the initial 2MASS+GALEX+SuperCOSMOS selection, and a low fraction of the initial CVs (11\%) and OBs (21\%), as listed in Table 2 (point 3). Absolute numbers after application of these selection criteria are also included in Table 2 (point 4).

\subsection{Spectral energy distribution fit}

Finally, we obtained a temperature estimate for the surviving targets by fitting their spectral energy distribution (SED). For this purpose, we employed the VO-tool VOSA ${ }^{7}$ (VO Sed Analyzer), which allows the user to query photometry from different catalogues, and compute $T_{\text {eff }}$ from comparison of the SEDs with those derived from a grid of theoretical spectra.

We used the 2mass and GaLEx photometry of the targets for the SED fit and any photometric data existent in other public archives. With only 2mass and GALEx photometry it is difficult to obtain reliable values of the effective temperature because 2MAss photometry is not sensitive to $T_{\text {eff }}$ changes within the typical temperature ranges for hot sds, i.e., large $T_{\text {eff }}$ variations may still yield good fits and because the GALEx magnitudes are highly dependent on reddening, and moderate errors in $E(B-V)$ translate into substantial errors in $T_{\text {eff }}$.

${ }^{7}$ http://svo.cab.inta-csic.es/theory/vosa/ 


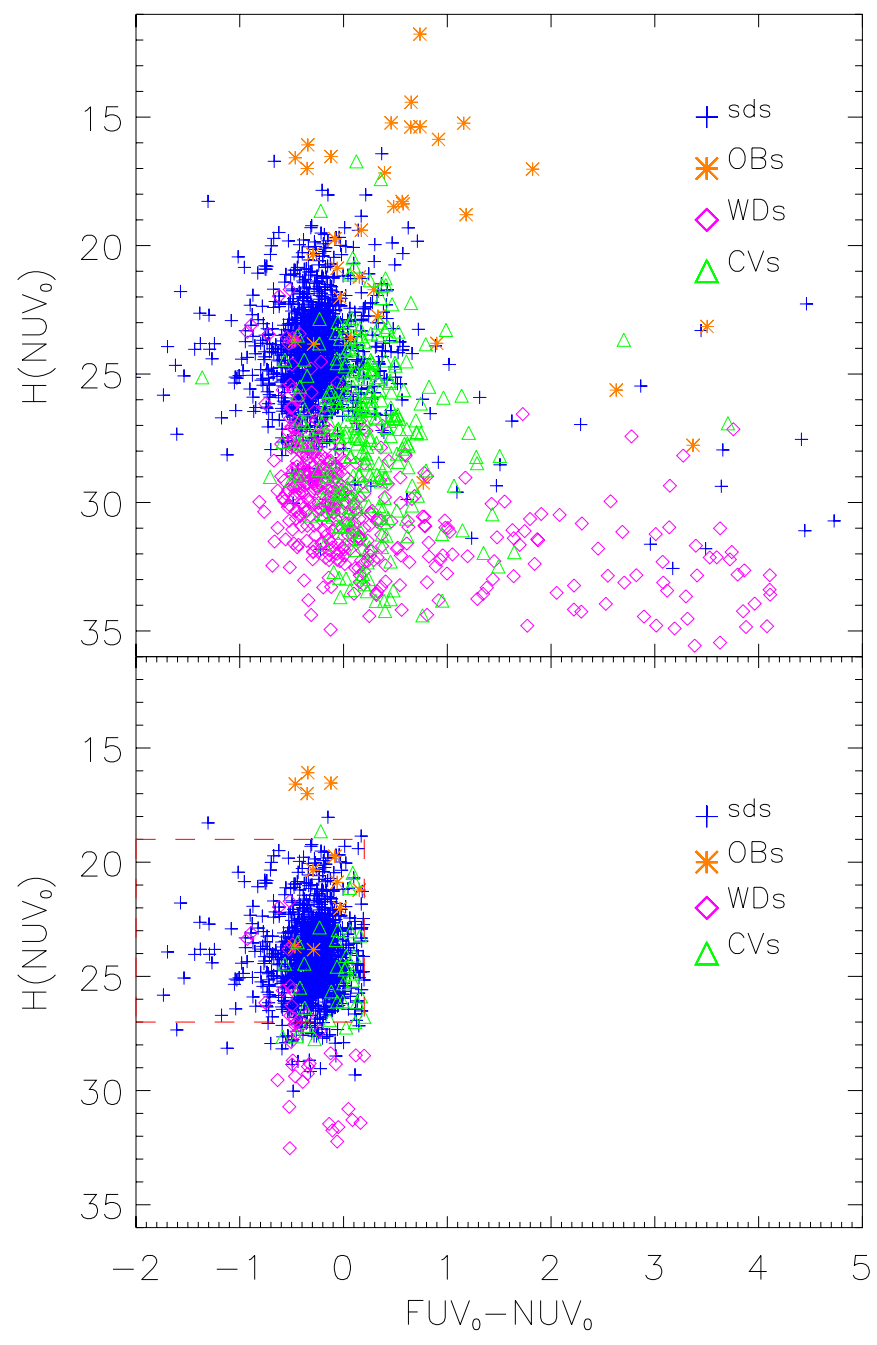

Fig. 3. Above: $H\left(N U V_{0}\right)$ against $\left(F U V_{0}-N U V_{0}\right)$ for the sample in Fig. 2. Notice the inverted $y$-axis scale. Below: Only objects fulfilling Eqs. (4) and (5) are plotted (see Sect. 3.2). The red dashed box gives $H\left(N U V_{0}\right)$ selection limits proposed in this work. See text and Table 2 for further details.

To solve this problem we used VOSA to obtain photometry from the SDSS/DR7 (Abazajian et al. 2009), UKIDSs (Lawrence et al. 2007), CMC-14 ${ }^{8}$ and TYCHO-2 (Høg et al. 2000) services for the objects fulfilling the two-colour and RPM criteria (see point 4 in Table 2). The magnitudes were then transformed into fluxes and deredened using the extinction law by Fitzpatrick (1999). The observed SED is fit with a grid of Kurucz model atmospheres (Castelli et al. 1997) with ranges $3500<T_{\text {eff }}<$ $50000 \mathrm{~K}, 2.5<\log g<5.5$. The temperature of the best fit for every object is represented in the histogram in Fig. 4. Note that this figure is shown in percentage for visibility reasons, given the low number of surviving objects other than hot sds.

We can see in Fig. 4 that WDs have high scattered temperatures, as expected because they are in different stages of the cooling phase. We note that five WDs have an M-dwarf companion, and for all of them we retrieve a $T_{\text {eff }}$ estimate below $40000 \mathrm{~K}$. Most of OBs and CVs have temperatures within $10-20 \mathrm{kK}$.

\footnotetext{
${ }^{8}$ http://www.ast.cam.ac.uk/cmt/cmc14.html
}

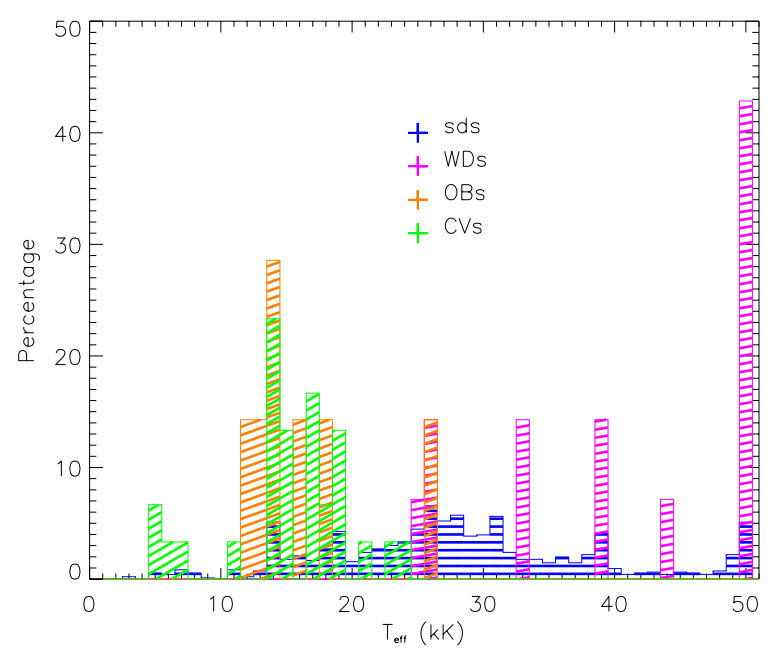

Fig. 4. Histogram showing the effective temperature distribution obtained with VOSA for the 960 hot sds (blue), 14 WDs (magenta), $30 \mathrm{CVs}$ (green) and 7 OBs (orange). See text for details.

When targets with the poorest goodness-of-fit are excluded from the plot, the location of OBs and CVs at $\sim 10-20 \mathrm{kK}$ is emphasized. Therefore, we can use the temperature estimate given by VOSA to discard a large part of $\mathrm{OB}$ and CV objects.

We chose to discard as hot sds candidates those objects with $T_{\text {eff }}<19000 \mathrm{~K}$ and an acceptable goodness-of-fit, while we mantain as candidates those with $T_{\text {eff }}>19000 \mathrm{~K}$ and those with poor fits, regardless of their $T_{\text {eff. }}$ The reason for this last choice is that O-type hot sds can have effective temperatures exceeding the Kurucz's upper limit of $50000 \mathrm{~K}$, and the fitting is expected to be poor for these hot objects ${ }^{9}$. The implementation in VOSA of a set of NLTE sdO models hotter than $50000 \mathrm{~K}$ is under investigation at the moment, and will eventually be used in a further extension of our study. A poor fit may be also obtained for composite hot sds.

The results are included in Table 2: $72 \%$ of the initial hot sds survive all imposed selection filters, while only $3 \%, 4 \%$, and $6 \%$ of the WDs, CVs, and OBs are selected as candidates, which corresponds to a very small fraction of contamination. It is difficult to asess the exact success rate, though, because the initial sample lists contain quite different numbers of objects.

\section{Application of the method}

In the previous section we designed a procedure to select a hot sds sample as pure as possible. We applied this method to two test sky regions to check its validity, and describe the results below.

\subsection{Test region A: Kepler FoV}

We tested our method in a region of about $420 \mathrm{deg}^{2}$, RA:(275, $305) \mathrm{deg}$, Dec: $(+33,+55) \mathrm{deg}$, which generously covers the Kepler $\mathrm{FoV}^{10}$ of $105 \mathrm{deg}^{2}$.

\footnotetext{
9 We checked, however, that for objects with $T_{\text {eff }}$ above the $50000 \mathrm{~K}$ Kurucz limit the VOSA $T_{\text {eff }}$ estimate is always above $19000 \mathrm{~K}$ although the obtained fit is poor. This ensures that any hot sdO will be kept by the selection procedure.

${ }^{10}$ http://kepler.nasa.gov/Science/targetField0fView/
} 
The Kepler FoV was chosen to test our method because extensive efforts are being made to select and classify suitable targets for long-term photometric monitoring. Within this framework, low-, intermediate-, and high-resolution spectra of a large number of targets in the FoV are being acquired by different groups (Uytterhoeven et al. 2010). Indeed, we use the Kepler test region to be able to confirm our hot sds candidate list, thanks to the above mentioned works, without the necessity of performing our own spectroscopic observations.

Our workflow comprised the following steps:

1. Cross-match: for each GALEx source within the field, we looked for all 2mass counterparts in a 4 arcsec circular region. If more than one 2mass counterpart was found, the GALEX source was removed ${ }^{11}$.

2. Data filtering

- we selected GALEx sources with $F U V$ and $N U V$ values brighter than the 5- $\sigma$ limiting magnitudes (19.9 and 20.8, respectively);

- we dereddened the Galex $(F U V, N U V)$ and 2mass $(K \mathrm{~s})$ photometry using Eqs. (1)-(3) to obtain the corrected $F U V_{0}, N U V_{0}$ and $K \mathrm{~s}_{0}$;

- we selected sources fulfilling $-4<\left(F U V_{0}-K \mathrm{~s}_{0}\right)<0.5$ and $-2<\left(F U V_{0}-N U V_{0}\right)<0.2$. This step left us with 90 candidates;

- we checked if the candidates were already in the catalogues used for defining the procedure (Sect. 3). After this step, 87 candidates remained $^{12}$;

- we retrieved the SuperCOSMOS proper motions for the candidates using a 5 arcsec search radius and applied the RPM selection criteria $\left(19<H\left(N U V_{0}\right)<27\right)$. This yielded 73 candidates (see Table 7 ).

3. SED fitting: for each candidate, we used VOSA to obtain its effective temperature from the theoretical model that best fitted the observed SED. In this particular field it was necessary to download the photometry available in the Kepler Input Catalog ${ }^{13}$ (KIC) to perform an acceptable SED reconstruction, because only the GALEX and 2MASs data are retrieved from VO services.

We thus restricted the analysis to those candidates with $g^{\prime} r^{\prime} i^{\prime} z^{\prime}$ SDSS photometry obtained during the Kepler preparatory programmes. This requisite limited our list to 21 objects, although we include in Table 7 the initial 73 candidates for general interest and indicate the KIC number for these 21 targets with additional photometry in the KIC.

From the VOSA analysis, we selected the good fits with $T_{\text {eff }}>19000 \mathrm{~K}$, and those which were not correctly fitted regardless of their temperature. After this step, we eventually ended up with 15 candidates. Table 3 gathers their photometry, while Fig. 5 includes the best SED fit for an example object.

Spectra were already available for all candidates in Table 3 (Østensen et al. 2010c, 2011), from which we benefit in checking the validity of our procedure. Thirteen of our candidates were

\footnotetext{
11 If more than one 2MAss match is found, the source is not further considered. Although the nearest one is supposed to be the correct infrared counterpart, we prefer to safely reject the candidate to avoid any missmatch and any UV contamination from the second close object, given the $\sim 4.5-6.0$ arcsec GALEX point-spread function.

12 Three objects are already catalogued: HS1844+5048 in The Subdwarf Database, V476Cyg in the Catalogue of Cataclysmic Variables and ALS10696 in the OB stars catalogue (Reed 2005).

${ }^{13}$ http://archive. stsci. edu/kepler/kic10/search.php
}

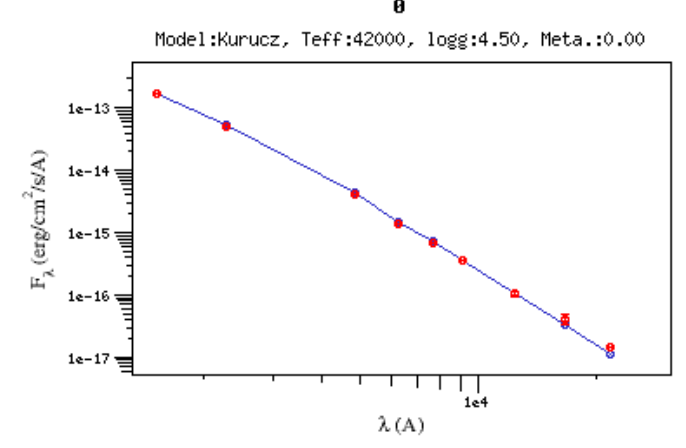

Fig. 5. Example of an SED fit performed by VOSA for object (18:43:07, $+42: 59: 18$ ) in the Kepler FoV (see Sect. 4.1 and Table 3).

classified as hot subdwarfs, the remaining objects are a mainsequence B star and a DA star, which confirmed the robustness and efficiency of our method (87\%).

Table 3 includes the spectral classification for the candidates, besides their corresponding KIC number. These 15 targets have all been already observed by Kepler, and Table 3 also indicates the survey cycle in which they were observed along with a brief description of their light curves.

\subsection{Test region B: RA: 210-240; Dec: 0-10}

We also applied our procedure to a region of $300 \mathrm{deg}^{2}$ : RA: (210, 240) deg, Dec: $(0,10)$ deg following the steps given in the previous section. We obtained a final list of 11 hot sds candidates, which are presented in Table 4 with their 2MAss, GALEX, and sDSs photometry, along with $T_{\text {eff }}$ estimated by VOSA and the spectral classification described below. For these objects we made use of Aladin ${ }^{14}$ to obtain the spectroscopic and catalogue data available in all Virtual Observatory services. The information is shown in Table 4 in the column "Comments".

Given that this region is covered by the Large Area Survey of UKIDSs ${ }^{15}$, we repeated the workflow in the same field using the UKIDSs Data Release 7 instead of 2MAss. Its large area coverage (7500 $\mathrm{deg}^{2}$ ) and depth (three magnitudes dimmer than 2MAss) makes it very adequate to search for non-catalogued, faint hot sds, which may not be catalogued by 2 MAss. The ukIDss search yielded 12 additional candidates, which are listed in Table 5 with the same information as in Table 4.

\subsubsection{Spectroscopic follow-up}

While all hot sd candidates from test region A were already classified, this is not the case for test region $\mathrm{B}$, where only a few objects are identified in the literature, as explained below. For this reason, we performed spectroscopic follow-up for candidates from test region $\mathrm{B}$, which we now describe.

Test region B: 2MASs-GaLEx: Five objects from Table 4 have an available SDSS spectrum. Four of them (J15104+0409, EGGR 491, J15513+0649, J15564+01131) are classified as hot subdwarfs in Østensen et al. (2010b), while a visual inspection of the fifth object $((15: 53: 33,+03: 44: 34)$, see Fig. 6) confirms

\footnotetext{
14 http://aladin.u-strasbg.fr/

15 http://www.ukidss.org/
} 
Table 3. List of candidates in the Kepler field-of-view.

\begin{tabular}{|c|c|c|c|c|c|c|c|c|c|c|c|c|}
\hline $\begin{array}{lc}\text { RA } & \text { Dec } \\
(\mathrm{J} 2000) & (\mathrm{J} 2000)\end{array}$ & $\begin{array}{ll}F U V & N U V\end{array}$ & $\overline{\overline{g^{\prime}}}$ & $\overline{r^{\prime}}$ & $\overline{i^{\prime}}$ & $\overline{z^{\prime}}$ & $\bar{J}$ & $\overline{\bar{H}}$ & $\overline{\bar{K}}$ & $\begin{array}{c}T_{\text {eff }} \\
\text { (VOSA) }\end{array}$ & Class. & $\begin{array}{c}\text { KIC } \\
\text { number }\end{array}$ & Com \\
\hline $18: 42: 42+44: 04: 06$ & 16.31716 .442 & 17.056 & 17.378 & 17.555 & 17.630 & 16.75 & 16.34 & 15.78 & $25000^{*}$ & $\mathrm{sdB}$ & 8142623 & $Q_{1}, R, a$ \\
\hline $18: 43: 07+42: 59: 18$ & 14.14914 .737 & 15.410 & 15.864 & 16.208 & 16.528 & 16.27 & 16.13 & $\mathrm{U}: 16.24$ & 42000 & $\mathrm{sdO}+\mathrm{dM}$ & 7335517 & $Q_{3}, R, b$ \\
\hline $18: 47: 14+47: 41: 47$ & 13.30513 .772 & 14.489 & 14.988 & 15.366 & 15.725 & 15.39 & 15.62 & 15.47 & 41000 & He-sdO & 10449976 & $Q_{3}, N, b$ \\
\hline $18: 50: 17+43: 58: 29$ & 16.50116 .387 & 16.353 & 16.649 & 16.996 & 17.115 & 16.71 & $\mathrm{U}: 17.15$ & U:17.00 & 23000 & B & 8077281 & $Q_{2}, I, a$ \\
\hline $19: 04: 35+48: 10: 22$ & 15.72616 .057 & 16.696 & 17.031 & 17.236 & 17.456 & 16.63 & U:16.34 & $\mathrm{U}: 17.11$ & 25000 & $\mathrm{sdB}$ & 10784623 & $Q_{5}, R, b$ \\
\hline $19: 05: 06+43: 18: 31$ & 14.20814 .391 & 15.058 & 15.516 & 15.894 & 16.193 & 15.81 & 16.06 & $\mathrm{U}: 16.23$ & 30000 & $\mathrm{sdB}$ & 7668647 & $Q_{3}, G, b$ \\
\hline $19: 08: 25+45: 08: 32$ & 15.26715 .518 & 16.283 & 16.605 & 16.755 & 16.819 & 16.17 & 15.78 & 15.36 & $26000^{*}$ & $\mathrm{sdB}$ & 8874184 & $Q_{4}, C, b$ \\
\hline $19: 08: 46+42: 38: 31$ & 13.98314 .490 & 15.146 & 15.595 & 15.964 & 16.295 & 15.90 & 16.05 & 16.02 & 35000 & $\mathrm{sdB}$ & 7104168 & $Q_{3}, N, b$ \\
\hline $19: 09: 33+46: 59: 04$ & 14.69515 .009 & 15.483 & 15.967 & 16.308 & 16.607 & 16.36 & $\mathrm{U}: 15.71$ & $\mathrm{U}: 16.61$ & 35000 & $\operatorname{sdB}$ & 10001893 & $Q_{3}, G, b$ \\
\hline $19: 10: 00+46: 40: 24$ & 14.11914 .496 & 14.572 & 14.583 & 14.613 & 14.672 & 13.93 & 13.80 & 13.73 & $18000^{*}$ & $\mathrm{sdO}+\mathrm{F} / \mathrm{G}$ & 9822180 & $Q_{2}, C, a$ \\
\hline $19: 14: 28+45: 39: 09$ & 15.00215 .339 & 15.895 & 16.174 & 16.297 & 16.423 & 16.02 & 15.57 & 15.68 & 26000 & $\mathrm{sdB}$ & 9211123 & $Q_{3}, N, b$ \\
\hline $19: 16: 12+47: 49: 16$ & 15.29115 .413 & 15.269 & 15.278 & 15.339 & 15.351 & 14.62 & 14.42 & 14.27 & $15000^{*}$ & $\mathrm{sdB}+\mathrm{F} / \mathrm{G}$ & 10593239 & $Q_{2,5}, C ?, a$ \\
\hline $51+49: 08: 48$ & 14.98114 .813 & 15.396 & 15.522 & 15.565 & 15.595 & 14.83 & 14.52 & 14.37 & $21000^{*}$ & $\mathrm{sdB}+\mathrm{F} / \mathrm{G}$ & 11350152 & $Q_{3}, C, b$ \\
\hline $2+48: 27: 23$ & 13.54413 .969 & 14.093 & 14.175 & 14.269 & 14.369 & 13.68 & 13.56 & 13.56 & $20000^{*}$ & $\mathrm{sdB}+\mathrm{F} / \mathrm{G}$ & 10982905 & $Q_{2}, C, a$ \\
\hline $19: 43: 44+50: 04: 38$ & 13.22813 .888 & 14.455 & 14.938 & 15.320 & 15.682 & 15.37 & 15.36 & 15.18 & $50000^{*}$ & DA & 11822535 & $Q_{2}, N, a$ \\
\hline
\end{tabular}

Notes. $F U V, N U V$ have been taken from the GaLEX archive; $g^{\prime}, r^{\prime}, i^{\prime}, z^{\prime}$ from the Kepler Input Catalogue and $J, H$, and $K$ from 2 Mass. "U" means upper limit in the 2mAss photometry. $T_{\text {eff }}$ is obtained from the best SED fit performed by VOSA. ${ }^{*}$ Indicates that a poor SED fit is obtained. KIC is the number from the Kepler input catalogue. ${ }^{\left(Q_{x}\right)}$ Kepler survey cycle of observation . Brief description of its light curve: ${ }^{(N)}$ no particular features; ${ }^{(R)}$ reflecting binary; ${ }^{(G)} \mathrm{g}$-mode pulsator; ${ }^{(I)}$ irregular variable; ${ }^{(C)}$ variability from the companion. Spectral classification: ${ }^{\left({ }^{(a)}\right.}$ from $\emptyset$ stensen et al. (2010c); ${ }^{(b)}$ from Østensen et al. (2011).

Table 4. List of candidates in the Test Region B obtained from the GALEX-2MAss-SuperC cross-match.

\begin{tabular}{|c|c|c|c|c|c|c|c|c|c|c|c|c|c|}
\hline $\begin{array}{l}\text { RA } \\
(\mathrm{J} 2000)\end{array}$ & $\begin{array}{c}\text { Dec } \\
(\mathrm{J} 2000)\end{array}$ & $\begin{array}{lll}F U V & N U V\end{array}$ & $u$ & $g$ & $r$ & $i$ & $z$ & $\bar{J}$ & $\overline{\bar{H}}$ & $\overline{\bar{K}}$ & $\begin{array}{c}T_{\text {eff }} \\
\text { (VOSA) }\end{array}$ & Class. & Comments \\
\hline $14: 14: 35$ & $+00: 12: 36$ & 14.37814 .770 & 15.389 & 15.725 & 16.240 & 16.585 & 16.921 & 16.46 & $\mathrm{U}: 16.37$ & $\mathrm{U}: 16.81$ & 48000 & & FBS 1412+004 \\
\hline $14: 23: 40$ & $+00: 10: 21$ & 16.07416 .669 & 17.469 & 17.854 & 18.065 & 17.914 & 17.683 & 16.19 & 15.57 & 15.58 & 44000 & & \\
\hline 14:58:06 & $+08: 51: 30$ & 13.88614 .282 & 14.380 & 14.349 & 14.839 & 15.160 & 15.474 & 15.14 & 15.11 & 15.01 & 23000 & $\mathrm{sdB}$ & \\
\hline $15: 10: 42$ & $+04: 09: 55$ & 15.83815 .962 & 16.541 & 16.810 & 17.249 & 17.498 & 17.692 & 16.72 & U:16.26 & U:16.45 & 31000 & sdOB & $\mathrm{J} 15104+0409^{a}$ \\
\hline $15: 16: 4$ & $+09: 26: 32$ & 15.85716 .285 & 16.854 & 17.019 & 17.295 & 17.452 & 17.641 & 16.57 & 16.26 & $\mathrm{U}: 16.06$ & 19000 & $s d B+F / G$ & \\
\hline $15: 35: 10$ & $+03: 11: 14$ & 14.02014 .727 & 24.109 & 15.636 & 16.148 & 16.517 & 16.851 & 16.48 & 16.42 & U:17.16 & $50000^{*}$ & DA & WD1532+033 \\
\hline $15: 43: 3$ & $+00: 12: 02$ & 16.20416 .437 & 16.708 & 16.726 & 17.027 & 17.169 & 17.325 & 16.71 & 16.26 & $\mathrm{U}: 15.44$ & 23000 & $\mathrm{sdB}$ & EGGR $491^{a}$ \\
\hline $15: 4$ & $+01: 32: 29$ & 16.29716 .690 & 16.901 & 16.673 & 16.580 & 16.525 & 16.512 & 15.62 & 15.10 & 14.99 & $21000^{*}$ & $s d B+F / G$ & \\
\hline $15: 51: 20$ & $+06: 49: 04$ & 15.36215 .591 & 15.987 & 15.891 & 15.936 & 15.949 & 15.988 & 15.26 & 14.98 & 14.90 & $8000^{*}$ & $\mathrm{sdOB}+\mathrm{X}$ & $\mathrm{J} 15513+0649^{a}$ \\
\hline $15: 53: 33$ & $+03: 44: 34$ & 16.53316 .785 & 16.618 & 16.688 & 16.828 & 16.913 & 17.018 & 16.29 & 16.03 & 15.54 & 24000 & He-sdOB & \\
\hline $15: 56: 28$ & $+01: 13: 35$ & 15.33815 .633 & 15.867 & 15.985 & 16.387 & 16.707 & 16.935 & 16.46 & 16.63 & $\mathrm{U}: 17.02$ & 29000 & sdB & $\mathrm{J} 15564+01131^{a}$ \\
\hline
\end{tabular}

Notes. $F U V, N U V$ have been taken from the GALEx archive; $u, g, r, i, z$ from the SDSS Data Release 7 and $J, H$, and $K$ from the 2Mass Point Source Catalogue. "U" means upper limit in the 2MAss photometry. $T_{\text {eff }}$ is obtained from the best SED fit performed by VOSA. ${ }^{(*)}$ Indicates that a poor SED fit is obtained. ${ }^{(a)}$ Catalogued in Østensen et al. (2010b); ${ }^{(b)}$ classified as DA by Koester et al. (2009).

Table 5. List of candidates in Test Region B obtained from the GALEX-UKIDSS-SuperC cross-match.

\begin{tabular}{|c|c|c|c|c|c|c|c|c|c|c|c|c|c|c|}
\hline $\begin{array}{l}\text { RA } \\
(\mathrm{J} 2000)\end{array}$ & $\begin{array}{c}\text { Dec } \\
(\mathrm{J} 2000)\end{array}$ & "FUV $\quad N U V$ & $u$ & $g$ & $r$ & $i$ & $z$ & $\overline{\bar{Y}}$ & $\overline{\bar{J}}$ & $\bar{H}$ & $\overline{\bar{K}}$ & $\begin{array}{c}T_{\text {eff }} \\
\text { (VOSA) }\end{array}$ & Class & "Comments \\
\hline $14: 15: 17$ & $+09: 49: 26$ & 17.48517 .968 & 18.765 & 19.150 & 19.493 & 19.317 & 18.958 & 18.267 & 17.804 & 17.2981 & 17.003 & $7200^{*}$ & & \\
\hline $14: 24: 37$ & $+02: 34: 19$ & 15.71216 .118 & 16.465 & 16.464 & 16.841 & 17.122 & 16.986 & 17.060 & 17.129 & 17.2201 & 17.245 & 23000 & DA & PG1422+028 ${ }^{a}$ \\
\hline $14: 34: 40$ & $+06: 07: 03$ & 15.98816 .524 & 17.288 & 17.714 & 18.175 & 18.258 & 18.140 & 17.508 & 17.142 & 16.6331 & 16.458 & 39000 & & \\
\hline $14: 39: 18$ & $+01: 02: 51$ & 16.32616 .285 & 16.391 & 16.367 & 16.769 & 17.060 & 17.340 & 16.968 & 17.054 & 17.1391 & 17.216 & 21000 & $\mathrm{sdB}$ & $\mathrm{J} 1439+0102^{b}$ \\
\hline $14: 4$ & $+03: 15: 06$ & 16.04916 .222 & 16.3 & 16.1 & 16.550 & 16.855 & 17.108 & 16.748 & 16.740 & 16.6981 & 16.809 & 20000 & & \\
\hline 15: & $+09: 13: 57$ & 16.19016 .354 & 16.9 & 17.2 & 17.763 & 18.119 & 18.489 & 18.117 & 18.220 & 18.4181 & 18.529 & 39000 & sdOB & \\
\hline 15 & $+09: 58: 51$ & 16.20716 .471 & 17. & 17 & 17.8 & 18.1 & 18.331 & 17.977 & 17.887 & 17.6341 & 17.625 & 31000 & sdOB & \\
\hline $15: 2$ & $+00: 16: 41$ & 15.02615 .603 & 16.1 & 16.5 & 17.066 & 17.436 & 17.787 & 17.489 & 17.514 & 17.6331 & 17.656 & 50000 & He-sdOB & $\mathrm{J} 1526+0016^{c}$ \\
\hline 15: & +08:02:37 & 16.92017 .223 & 17.7 & 17.839 & 18.194 & 18.397 & 18.679 & 18.161 & 18.195 & 18.1181 & 17.883 & 19000 & & \\
\hline $15: 2$ & $+09: 31: 44$ & 15.05915 .374 & 15.920 & 16.161 & 16.664 & 17.030 & 17.340 & 17.046 & 17.148 & 17.2231 & 17.345 & 34000 & sdOB & \\
\hline $15 \cdot 3$ & $+06: 56: 52$ & 7915.656 & 16.0 & 16.173 & 16.643 & 16.949 & 17.266 & 16.921 & 17.012 & 17.0201 & 17.070 & 27000 & & \\
\hline $15: 3$ & $+03: 08: 13$ & 15.71815 .932 & 16.449 & 16.686 & 17.169 & 17.507 & 17.824 & 17.488 & 17.572 & 17.6451 & 17.796 & 34000 & & \\
\hline
\end{tabular}

Notes. $F U V, N U V$ have been taken from the GALEx archive; $u, g, r, i, z$ from the SDSS Data Release 7 and $Y, J, H$, and $K$ from the uKIDSs Large Area Survey (DR7). $T_{\text {eff }}$ is obtained from the best SED fit performed by VOSA. ${ }^{(*)}$ Indicates that a poor SED fit is obtained. ${ }^{(a)}$ Catalogued in McCook \& Sion (1999); ${ }^{(b)}$ classified as sdB in Eisenstein et al. (2006); ${ }^{(c)}$ classified as sdO in Eisenstein et al. (2006). 


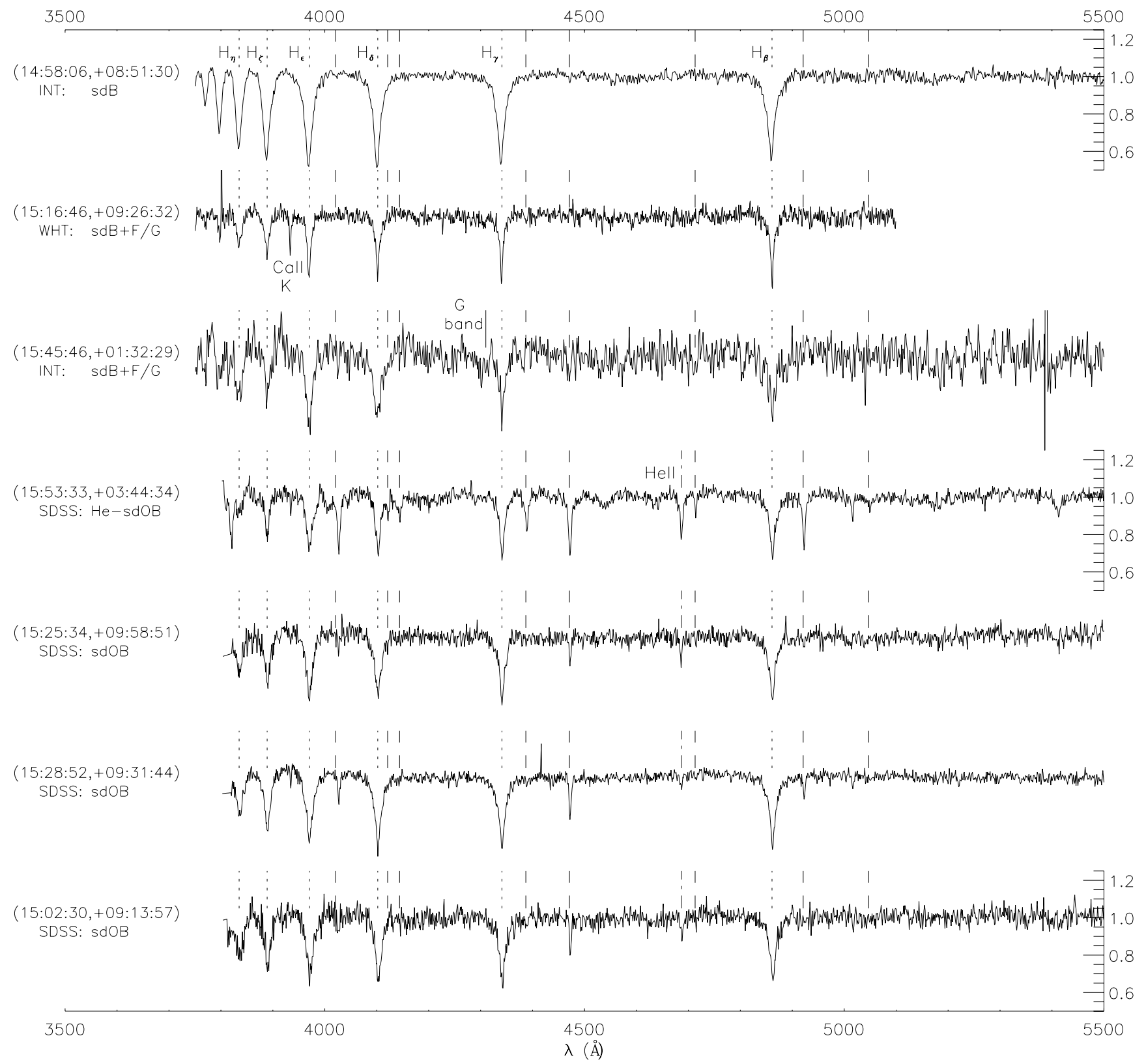

Fig. 6. Follow-up spectroscopy gathered at the INT and WHT for some candidates of Table 4, and SDSS spectra that were available for some others from Tables 4 and 5 (last four panels). The first Balmer lines are indicated as dotted vertical lines; dashed vertical lines indicate the HeI spectral lines; HeII4686 is marked with a dashed-dotted line; the G-band is also indicated when present.

that this is a hot subdwarf as well. On the other hand, Koester et al. (2009) classify WD $1532+033$ as a DA object.

Follow-up spectroscopy of three additional objects from Table 4 could be gathered at the Isaac Newton Telescope (INT) and the William Herschel Telescope (WHT) in La Palma (Spain) as a filling programme. The IDS spectrograph mounted on the INT was used with the R400B grating, which provided a resolution of $R \approx 1400$ and an effective wavelength coverage $\lambda \simeq 3100-6700 \AA$. The ISIS spectrograph was used the WHT with the grating R300B on the blue arm $(R \approx 1600, \lambda \bumpeq$ 3100-5300 ̊). Standard IRAF packages were used for the data reduction, which included bias subtraction, flatfield correction and wavelength calibration. The extracted spectra were normalized to obtain a raw spectral classification to check the success rate of our methodology. The normalized spectra of these objects are included in Fig. 6.

The first object $(14: 58: 06,+08: 51: 30)$ displays a typical sdB spectrum, with only broad Balmer lines up to a low series number. The CaII $\mathrm{H}$ and $\mathrm{K}$ lines may indicate an sdB star with an F or G type companion, but may also be caused by a significant amount of dust along the line of sight. The CaII $\mathrm{H}$ line, when present, deepens the $\mathrm{H}_{\epsilon}$ line, and is often used as an indicator of $\mathrm{sdB}+\mathrm{F} / \mathrm{G}$ composites. The second object in Fig. 6, (15:16:46, $+09: 26: 32$ ), seems to be a case of such an $\mathrm{sdB}+\mathrm{F} / \mathrm{G}$ composite. 
The spectrum of $(15: 45: 46,+01: 32: 29)$ is noisier, but the G-band together with the deep $\mathrm{H}_{\epsilon}$ line hints towards a composite nature for this star, too. When taken together with the distinctive red excess seen in the SED (Fig. 7), the composite nature of this object is quite certain. Modelling this object with two Kurucz components provides an excellent fit, as illustrated in the lower panel of Fig. 7. The optimum fit is achieved for a hot+cool pair with temperatures $30000+5000 \mathrm{~K}$ and a radius ratio $R_{\text {cool }} / R_{\text {hot }} \simeq 8$.

The SDSS spectrum of object $(15: 53: 33,+03: 44: 34)$ is also included in Fig. 6, which is identified as a He-sdOB based on the presence of Balmer, HeI, and HeII lines.

We use the same classification scheme as in Østensen et al. (2010c), in which a distinction is made between the common He-sdOB stars showing HeI and HeII with almost equal depth, and the hotter and more scarce He-sdOs, with predominantly HeII lines.

Test region B: UKIDSS-GALEX: We were unable to perform any follow-up spectroscopy for candidates in Table 5 owing to their faintness. However, one object is classified as WD DA by McCook \& Sion (1999) (PG1422+028), and two objects are identified as hot sds by Eisenstein et al. (2006) (J1439+0102: sdB; J1526+0016: sdO). Moreover, three other targets have an SDSS spectrum: $(15: 02: 30,+09: 13: 57),(15: 25: 34,+09: 58: 51)$, $(15: 28: 52,+09: 31: 44)$, and we also include them in Fig. 6 at the bottom. The three have very similar spectra with several HeI lines: $4027,4471 \AA$ (and $4922 \AA$ in the case of $(15: 28: 52$, $+09: 31: 44)$ ) plus the HeII4686 line (only traces for $(15: 28: 52$, $+09: 31: 44)$ ) in addition to the Balmer series. We classify all of them as sdOB objects, as indicated in Table 5.

\section{Binary hot subdwarfs in the sample}

In the lists of candidates of both test region $\mathrm{A}$ and $\mathrm{B}$ (Tables 3-5), we indicated with an asterisk those targets for which a poor SED fit is obtained. A poor fit is expected to occur for objects hotter than $T_{\text {eff }}>50000 \mathrm{~K}$ (the hot end of the Kurucz grid used), as this seems to happen for the white dwarfs $(19: 43: 44,+50: 04: 38)$ in Table 3 and $(15: 35: 10,+03: 11: 14)$ in Table 4.

If a target is in a binary system with a cool companion, its impact at long wavelengths can also cause a poor SED fit. We encountered nine such cases with a more or less clear infrared excess that may be caused by a red companion. For these cases, we fitted the spectral distribution to a combination of two Kurucz model atmospheres. In Fig. 7 we can see how a two-component fit (lower panel) yields a much better match to the observed spectral distribution compared to a single-component fit (upper panel) for object $(15: 45: 46,+01: 32: 29)$. The temperatures of the best hot+cool pair for the nine cases are included in Table 6 . The two-component fit did not improve the single-component one performed by VOSA for only two targets; for these no temperatures are included in the table and further analyses are planned for these two objects.

\section{Conclusions}

We developed a methodology to find new uncatalogued hot subdwarfs through using large databases such as GALEX, 2MAss, UKIDSs, and SuperCOSMOS. The VO tools helped to handle the different queries and the large output list of candidates. We tested the methodology with bona-fide input objects from the literature, which are spectroscopically classified in the catalogues listed in Sect. 3.
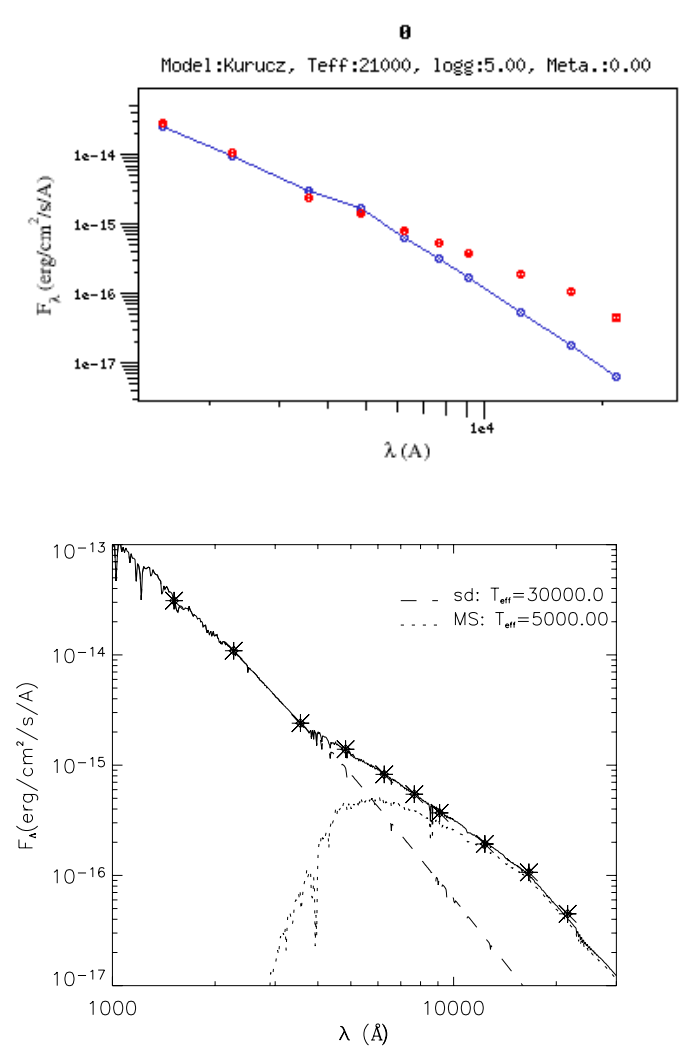

Fig. 7. Upper: best VOSA SED fit for the object $(15: 45: 46,+01: 32: 29)$. It displays the G-band in the spectrum (see Fig. 6), and an infrared excess in the spectral distribution. Bottom: best fit obtained considering two Kurucz components.

Table 6. Two-component SED decomposition for objects with a poor fit in Tables 3-5.

\begin{tabular}{lcc}
\hline \hline Object & $T_{\text {eff }}$ hot & $T_{\text {eff }}$ cool \\
\hline 18:42:42+44:04:06 & - & - \\
19:08:25+45:08:32 & - & - \\
19:10:00 +46:40:24 & 37000 & 6250 \\
$19: 16: 12+47: 49: 16$ & 22000 & 5250 \\
$19: 26: 51+49: 08: 48$ & 28000 & 5000 \\
$19: 40: 32+48: 27: 23$ & 31000 & 5750 \\
$15: 45: 46+01: 32: 29$ & 30000 & 5000 \\
$15: 51: 20+06: 49: 04$ & 29000 & 5500 \\
$14: 15: 17+09: 49: 26$ & 29000 & 5500 \\
\hline
\end{tabular}

The 2MASs-GALEX photometry combination was first used to separate blue objects from redder ones. A selection criterion given by Eqs. (4) and (5) effectively retrieves hot sd candidates, along with other UV-excess objects: mainly white dwarfs, cataclysmic variables, and main-sequence OB stars. This first filtering leaves $87 \%$ of the input hot sds, but only $10 \%, 13 \%$ and $33 \%$ of the input WDs, CVs, and OB stars. Using reduced proper motions, the hot sd selection further improves, mainly because very few WDs fulfil the adopted proper motion criterion (see Table 2).

Moreover, the Virtual Observatory SED Analyzer (VOSA) was used to obtain a rough guess of temperatures for the candidates. These are computed by fitting Kurucz model atmospheres to the spectral energy distribution, and are constructed using all the available photometry for every target. We adopted 
R. Oreiro et al.: A search for new hot subdwarf stars by means of Virtual Observatory tools

Table 7. List of the 73 candidates in and around the Kepler field-of-view that fulfil the photometric and proper motion criteria.

\begin{tabular}{|c|c|c|c|c|c|c|c|c|c|c|c|c|c|c|c|}
\hline $\begin{array}{c}\text { RA } \\
(\mathrm{J} 2000)\end{array}$ & $\begin{array}{c}\text { Dec } \\
(\mathrm{J} 2000)\end{array}$ & $F U V$ & $\overline{N U V}$ & $J$ & $\bar{H}$ & $\bar{K}$ & $\begin{array}{c}\text { KIC } \\
\text { number }\end{array}$ & $\begin{array}{c}\text { RA } \\
(\mathrm{J} 2000)\end{array}$ & $\begin{array}{c}\text { Dec } \\
(\mathrm{J} 2000)\end{array}$ & $\overline{F U V}$ & $N U V$ & $J$ & $\bar{H}$ & $\bar{K}$ & $\begin{array}{c}\text { KIC } \\
\text { number }\end{array}$ \\
\hline 18:19:48 & $+33: 22: 09$ & 15.704 & 15.745 & 17.02 & 17.04 & 16.48 & & $18: 50: 46$ & $+51: 07: 38$ & 14.416 & 14.134 & 14.01 & 14.10 & 14.17 & \\
\hline $18: 20: 55$ & $+33: 18: 47$ & 16.137 & 16.291 & 17.03 & 16.63 & 17.47 & & $18: 57: 58$ & $+44: 40: 57$ & 16.300 & 16.454 & 15.79 & 15.63 & 15.40 & 8544347 \\
\hline $18: 20: 58$ & $+37: 07: 07$ & 14.441 & 14.658 & 16.49 & 15.89 & 16.53 & & $19: 03: 44$ & $+47: 24: 39$ & 14.991 & 15.303 & 15.45 & 14.94 & 14.93 & \\
\hline $18: 21: 10$ & $+34: 46: 45$ & 13.062 & 14.152 & 15.29 & 15.50 & 15.47 & & $19: 04: 35$ & $+48: 10: 22$ & 15.726 & 16.057 & 16.63 & 16.34 & 17.11 & $10784623^{*}$ \\
\hline $18: 21: 50$ & $+41: 51: 56$ & 16.438 & 16.870 & 16.79 & 16.80 & 15.87 & & 19:05:06 & $+43: 18: 31$ & 14.208 & 14.391 & 15.81 & 16.06 & 16.23 & $7668647^{*}$ \\
\hline $18: 22: 43$ & $+43: 20: 37$ & 12.865 & 13.110 & 14.40 & 14.52 & 14.50 & & 19:05:20 & $+44: 57: 59$ & 17.545 & 17.594 & 6.41 & 15.93 & 17.28 & 8741434 \\
\hline $18: 23: 10$ & $+33: 33: 55$ & 14.461 & 14.451 & 15.66 & 15.56 & 15.99 & & 19:08:25 & $+45: 08: 32$ & 15.267 & 15.518 & 16.17 & 15.78 & 15.36 & $8874184^{*}$ \\
\hline $18: 23: 57$ & $+41: 29: 14$ & 13.682 & 13.653 & 15.16 & 15.29 & 15.64 & & 19:08:46 & $+42: 38: 32$ & 13.983 & 14.490 & 15.90 & 16.05 & 16.02 & $7104168^{*}$ \\
\hline $18: 24: 00$ & $+51: 55: 25$ & 14.362 & 14.801 & 16.25 & 16.08 & 15.94 & & 19:09:20 & $+45: 40: 57$ & 14.295 & 14.622 & 14.72 & 14.87 & 14.88 & \\
\hline $18: 24: 08$ & $+35: 16: 19$ & 14.767 & 15.129 & 15.41 & 15.11 & 14.97 & & 19:09:34 & $+46: 59: 04$ & 14.695 & 15.009 & 16.36 & 15.71 & 16.61 & $10001893^{*}$ \\
\hline $18: 24: 34$ & $+38: 00: 54$ & 15.723 & 15.811 & 16.39 & 16.94 & 15.74 & & 19:10:00 & $+46: 40: 25$ & 14.119 & 14.496 & 13.93 & 13.80 & 13.73 & $9822180^{*}$ \\
\hline $18: 24: 44$ & $+35: 30: 42$ & 14.218 & 14.603 & 16.83 & 16.64 & 17.33 & & $19: 10: 24$ & $+47: 09: 45$ & 11.796 & 12.368 & 11.31 & 11.45 & 11.47 & 10130954 \\
\hline $18: 24: 49$ & $+38: 51: 38$ & 14.516 & 14.947 & 16.15 & 16.31 & 15.67 & & $19: 14: 28$ & $+45: 39: 11$ & 15.002 & 15.339 & 16.02 & 15.57 & 15.68 & $9211123^{*}$ \\
\hline $18: 25: 19$ & $+40: 33: 34$ & 15.010 & 15.139 & 16.26 & 16.43 & 16.76 & & $19: 15: 08$ & $+47: 54: 20$ & 13.617 & 14. & 13.30 & 13.33 & 13.36 & 10658302 \\
\hline $18: 26: 22$ & $+32: 51: 08$ & 14.236 & 14.637 & 15.54 & 16.18 & 15.25 & & $19: 16: 12$ & $+47: 49: 16$ & 15.291 & 15.413 & 14.62 & 14.42 & 14.27 & $10593239^{*}$ \\
\hline $18: 26: 37$ & $+34: 37: 26$ & 14.578 & 14.714 & 16.40 & 16.46 & 17.34 & & $19: 20: 03$ & $+49: 15: 33$ & 15.392 & 15.5 & 16.76 & 16.29 & 15.84 & \\
\hline $18: 27: 45$ & $+37: 09: 31$ & 16.518 & 16.700 & 16.48 & 16.42 & 16.06 & & 19:20:18 & $+48: 06: 21$ & 15.655 & 15.873 & 16.74 & 17.19 & 16.25 & \\
\hline $18: 27: 55$ & $+36: 22: 08$ & 14.688 & 14.938 & 16.60 & 15.86 & 16.13 & & $19: 20: 36$ & $+49: 03: 16$ & 14.275 & 14.500 & 13.50 & 13.49 & 13.54 & 11293898 \\
\hline $18: 28: 50$ & $+34: 36: 50$ & 14.217 & 14.627 & 16.55 & 15.70 & 16.14 & & $19: 26: 52$ & $+49: 08: 49$ & 14.981 & 14.813 & 14.83 & 14.52 & 14.37 & $11350152^{*}$ \\
\hline $18: 32: 21$ & $+55: 03: 00$ & 15.117 & 15.713 & 15.50 & 14.85 & 14.83 & & $19: 30: 49$ & $+54: 21: 28$ & 16.309 & 16.531 & 16.62 & 16.28 & 16.57 & \\
\hline $18: 33: 44$ & $+43: 01: 06$ & 15.482 & 15.580 & 16.49 & 16.40 & 16.71 & & $19: 36: 33$ & $+52: 45: 19$ & 16.609 & 17.221 & 16.74 & 15.97 & 15.42 & \\
\hline $18: 34: 54$ & $+44: 49: 17$ & 14.607 & 14.726 & 13.85 & 13.63 & 13.51 & & $19: 40: 32$ & $+48: 27: 24$ & 13.544 & 13.969 & 13.68 & 13.56 & 13.56 & $10982905^{*}$ \\
\hline $18: 35: 17$ & $+43: 27: 30$ & 14.230 & 14.219 & 14.26 & 14.22 & 14.14 & & $19: 43: 44$ & $+50: 04: 39$ & 13.228 & 13.888 & 15.37 & 15.36 & 15.18 & $11822535^{*}$ \\
\hline $18: 36: 21$ & $+40: 59: 38$ & 14.974 & 15.221 & 13.98 & 13.87 & 13.79 & & $19: 44: 43$ & $+54: 49: 43$ & 15.654 & 15.748 & 16.11 & 16.01 & 15.83 & \\
\hline $18: 36: 34$ & $+53: 16: 57$ & 12.861 & 13.589 & 12.74 & 12.79 & 12.84 & & $19: 50: 24$ & $+50: 09: 00$ & 15.583 & 15.587 & 13.61 & 13.60 & 13.68 & \\
\hline 18:36:42 & $+41: 30: 46$ & 15.252 & 15.477 & 15.08 & 14.91 & 14.97 & & 19:53:04 & $+49: 49: 34$ & 15.581 & 15.923 & 16.32 & 16.23 & 15.62 & \\
\hline 18:39:49 & $+53: 00: 04$ & 14.797 & 15.050 & 16.67 & 16.01 & 15.98 & & $19: 53: 42$ & $+49: 59: 45$ & 14.713 & 15.329 & 16.47 & 16.27 & 16.47 & \\
\hline 18:40:21 & $+41: 43: 15$ & 15.376 & 15.386 & 15.24 & 15.24 & 15.11 & & $19: 54: 52$ & $+48: 22: 29$ & 15.014 & 14.920 & 13.95 & 13.96 & 13.99 & 10937527 \\
\hline $18: 42: 03$ & $+45: 31: 59$ & 14.883 & 14.842 & 14.56 & 14.62 & 14.55 & & $19: 56: 48$ & $+53: 12: 17$ & 15.413 & 15.941 & 15.27 & 14.76 & 14.54 & \\
\hline $18: 42: 42$ & $+44: 04: 05$ & 16.317 & 16.442 & 16.75 & 16.34 & 15.78 & $8142623^{*}$ & $19: 57: 28$ & $+53: 32: 03$ & 13.989 & 14.650 & 14.47 & 14.19 & 14.02 & \\
\hline 18:43:07 & $+42: 59: 18$ & 14.149 & 14.737 & 16.27 & 16.13 & 16.24 & $7335517^{*}$ & 20:00:01 & $+54: 09: 03$ & 15.202 & 15.155 & 12.55 & 12.54 & 12.52 & \\
\hline $18: 43: 56$ & $+45: 37: 57$ & 15.009 & 15.204 & 16.75 & 16.90 & 16.79 & & $20: 01: 54$ & $+49: 03: 54$ & 15.119 & 15.456 & 16.44 & 15.74 & 17.05 & \\
\hline $18: 47: 14$ & $+47: 41: 47$ & 13.305 & 13.772 & 15.39 & 15.62 & 15.47 & $10449976^{*}$ & $20: 06: 33$ & $+48: 33: 29$ & 12.383 & 13.752 & 9.14 & 9.11 & 9.08 & \\
\hline $18: 47: 46$ & $+50: 41: 35$ & 13.877 & 14.061 & 15.24 & 15.45 & 15.94 & & 20:07:39 & $+54: 45: 16$ & 17.424 & 17.667 & 16.81 & 16.29 & 15.84 & \\
\hline 18:49:15 & $+51: 16: 05$ & 13.792 & 13.941 & 15.54 & 15.58 & 15.09 & & $20: 09: 34$ & $+55: 05: 25$ & 18.663 & 18.5 & 15.76 & 15.52 & 15.44 & \\
\hline 18:50:05 & $+50: 24: 22$ & 15.206 & 15.315 & 14.60 & 14.52 & 14.39 & & $20: 11: 52$ & $+54: 50: 11$ & 14.201 & 13.914 & 10.11 & 10.05 & 10.02 & \\
\hline 18:50:17 & $+43: 58: 29$ & 16.501 & 16.387 & 16.71 & 17.15 & 17.00 & $8077281^{*}$ & & & & & & & & \\
\hline
\end{tabular}

Notes. $F U V, N U V$ have been taken from the GaLEX archive and $J, H$, and $K$ from 2Mass. The KIC number for 21 objects with additional photometry is included. ${ }^{(*)}$ Objects meeting all the selection criteria, detailed in Table 3.

as a hot sds threshold objects with $T_{\text {eff }}>19000 \mathrm{~K}$. This criterion is specially useful to filter out CVs and OB stars, because generally lower temperatures are obtained by VOSA for these objects (see Fig. 4). Targets with a poor fit were retained as good candidates regardless of their temperature estimate, given that a poor fit may be obtained for stars that show infrared excess that is indicative of a cool companion, or for sdOs of high temperature $\left(T_{\text {eff }}>50000 \mathrm{~K}\right)$.

After all these filters were imposed, $72 \%$ of the initial hot sds remained, while, on the other hand, only a low fraction of other spectral types meet the criteria adopted: $3 \%, 4 \%$ and $6 \%$ of the initial WDs, CVs, and OBs respectively.

We applied this strategy to two test regions: a $\sim 420 \mathrm{deg}^{2}$ region centred on the Kepler satellite field-of-view (test region A), and the other at RA: 210-240, Dec: 0-10 (test region B). In the
Kepler FoV, 73 objects fulfil the colour and proper motion criteria. However, only 21 have additional KIC $g^{\prime} r^{\prime} i^{\prime} z^{\prime}$ photometry besides 2MASs and GALEX, which is necessary to perform an acceptable SED fit. Thirteen of them have temperature estimates above $19000 \mathrm{~K}$ and two are retained because of their poor fit (see Table 3). Thanks to the ground-based support of the Kepler mission, spectroscopic follow-up of all the candidates was available. Thirteen candidates are confirmed to be hot sds, with only two targets contaminating the sample: a main-sequence B star, and a white dwarf.

For test region B, we applied the same methodology and obtained a list of 11 candidates (see Table 4), three of which were retained owing to a poor SED fit. Four of these objects have been recently classified as hot sds by $\emptyset$ stensen et al. (2010b), a fifth target is classified as DA by Koester et al. (2009), and 
a sixth candidate $(15: 53: 33,+03: 44: 34)$ has a spectrum in the SDSS database, which identifies the object as a hot sd. Followup spectroscopy was acquired for three other objects at the INT and WHT (La Palma). The classification is included in Table 4.

Furthermore, we repeated the same procedure for test region $\mathrm{B}$, but using UKIDss instead of the 2mass database. Twelve candidates are proposed, of which two are classified as hot sds by Eisenstein et al. (2006), one is labelled as DA by McCook \& Sion (1999) and three are identified as hot sds in this paper based on available Sloan spectra (see Fig. 6).

In total, we proposed 38 candidates, of which 30 could be spectroscopically classified, and 26 of them were confirmed to be hot sds. The success rate is thus $87 \%$. This high percentage confirms the suitability of our methodology to discover new hot subdwarfs.

Former surveys described in Sect. 2 aimed at finding faint blue stars in general and not only hot subdwarfs in particular. Thus, it is intrinsically improper to compare our finding rate with the percentage of hot sds found in these works. However, they rate a maximum of $53 \%$ of hot sds and demonstrate the difficulty of this task due to the photometric (and spectroscopic) similitudes among blue objects.

$\emptyset$ stensen et al. (2010c, 2011) compile the variety of methods used by several teams to obtain uncatalogued blue compact targets within the Kepler FoV. Most of the methods are based only on photometric colours and do not particularly intend to discern white dwarfs from hot sds, because both classes are of interest from a seismological point of view. Their success rate ranges from poor, when only 2Mass colours are considered, to actually very good, when using SDSS filters. The complete sample listed in Østensen et al. (2010c, 2011) is formed of $68 \mathrm{sds}$, 17 WDs, 14 Bs, 2 PNN, 3 CVs and 6 other main-sequence stars. Comparing this with the number of hot sds candidates proposed in this work, only 19 of the 68 hot sds in Østensen et al. (2010c, 2011) possess all the necessary data required by our methodology (2MASs, GALEX and proper motion), 17 of which fulfil all our selection criteria. We retrieved 13 of them; the GALEx photometry of the remaining four hot sds is obtained from the guest investigator survey, which was not public at the time our search was performed, and thus these targets are not listed as candidates in Table 3. This serves as a cross-check that a low fraction of hot sds are rejected by our search method, as expected from the tests made in Sect. 3, and that we will be able to increase our detections as the sky is better covered by the large surveys we make use of.

The use of proper-motion data was particularly suitable for finding WDs, as described in Østensen et al. (2010c, 2011). Proper-motion information was also useful to spot WDs and hot sds by Jiménez-Esteban et al. (2011), whose objective is the identification of blue high proper-motion objects. Combined photometric indices and proper-motion data are also employed by Vennes et al. (2011) with the intention of finding new white dwarfs. Their bright sample of candidates contains one single (and already known) WD, plus 15 hot sds (6 already catalogued), 29 main-sequence B stars, and five other blue objects of a different nature.

Encouraged by the results in the two pilot regions and taking advantage of the Virtual Observatory capabilities, we initiated a systematic search for hot subdwarf stars in the Milky Way, the results of which will be published in a forthcoming paper.

Acknowledgements. This research has made use of the Spanish Virtual Observatory supported from the Spanish MEC through grant AyA2008-02156. R.O., C.R.-L. and A.U. acknowledge financial support from the Spanish Ministry of Science and Innovation (MICINN) through grant AYA 2009-14648-02 and from the Xunta de Galicia through grants INCITE09 E1R312096ES and INCITE09 312191PR, all of them partially supported by E.U. FEDER funds. C.R.L. acknowledges an Ángeles Alvariño contract from the Xunta de Galicia and financial support provided from the Annie Jump Cannon grant of the Department of Physics and Astronomy of the University of Delaware.

R.O. thanks J. Gutiérrez-Soto for a careful reading of the manuscript and fruitful discussions. The research leading to these results has received funding from the European Research Council under the European Community's Seventh Framework Programme (FP7/2007-2013)/ERC grant agreement No. 227224 (PROSPERITY), as well as from the Research Council of K.U. Leuven grant agreement FOA/2008/04.

\section{References}

Abazajian, K. N., Adelman-McCarthy, J. K., Agüeros, M. A., et al. 2009, ApJS, 182,543

Altmann, M., Edelmann, H., \& de Boer, K. S. 2004, A\&A, 414, 181

Auvergne, M., Bodin, P., Boisnard, L., et al. 2009, A\&A, 506, 411

Bauer, F., \& Husfeld, D. 1995, A\&A, 300, 481

Borucki, W. J., Koch, D., Basri, G., et al. 2010, Science, 327, 977

Cardelli, J. A., Clayton, G. C., \& Mathis, J. S. 1989, ApJ, 345, 245

Castelli, F., Gratton, R. G., \& Kurucz, R. L. 1997, A\&A, 318, 841

Charpinet, S., Green, E. M., Baglin, A., et al. 2010, A\&A, 516, L6

Chavira, E. 1958, Boletin de los Observatorios Tonantzintla y Tacubaya, 2, 15

D’Cruz, N. L., Dorman, B., Rood, R. T., \& O'Connell, R. W. 1996, ApJ, 466, 359

Demers, S., Beland, S., Kibblewhite, E. J., Irwin, M. J., \& Nithakorn, D. S. 1986, AJ, 92, 878

Dorman, B., Rood, R. T., \& O’Connell, R. W. 1993, ApJ, 419, 596

Downes, R. A. 1986, ApJS, 61, 569

Downes, R. A., Webbink, R. F., Shara, M. M., et al. 2006, VizieR Online Data Catalog, 5123, 0

Dreizler, S., Heber, U., Werner, K., Moehler, S., \& de Boer, K. S. 1990, A\&A, 235, 234

Edelmann, H., Heber, U., Hagen, H., et al. 2003, A\&A, 400, 939

Eisenstein, D. J., Liebert, J., Harris, H. C., et al. 2006, ApJS, 167, 40

Feige, J. 1958, ApJ, 128, 267

Fitzpatrick, E. L. 1999, PASP, 111, 63

Fontaine, G., Brassard, P., Green, E. M., et al. 2008, A\&A, 486, L39

For, B., Green, E. M., Fontaine, G., et al. 2010, ApJ, 708, 253

Green, R. F., Schmidt, M., \& Liebert, J. 1986, ApJS, 61, 305

Greenstein, J. L., 1960, Stellar atmospheres, 890

Greenstein, J. L. 1966, ApJ, 144, 496

Hagen, H., Groote, D., Engels, D., \& Reimers, D. 1995, A\&AS, 111, 195

Hambly, N. C., Davenhall, A. C., Irwin, M. J., \& MacGillivray, H. T. 2001 MNRAS, 326, 1315

Han, Z., Podsiadlowski, P., Maxted, P. F. L., Marsh, T. R., \& Ivanova, N. 2002, MNRAS, 336, 449

Han, Z., Podsiadlowski, P., Maxted, P. F. L., \& Marsh, T. R. 2003, MNRAS, 341 669

Haro, G., \& Luyten, W. J. 1962, Boletin de los Observatorios Tonantzintla y Tacubaya, 3, 37

Heber, U. 1986, A\&A, 155, 33

Heber, U., Jordan, S., \& Weidemann, V. 1991, in White Dwarfs, ed. G. Vauclair, \& E. Sion, NATO ASIC Proc., 336, 109

Høg, E., Fabricius, C., Makarov, V. V., et al. 2000, A\&A, 355, L27

Humason, M. L., \& Zwicky, F. 1947, ApJ, 105, 85

Iriarte, B., \& Chavira, E. 1957, Boletin de los Observatorios Tonantzintla y Tacubaya, 2, 3

Jiménez-Esteban, F. M., Caballero, J. A., \& Solano, E. 2011, A\&A, 525, A29

Kilkenny, D., Heber, U., \& Drilling, J. S. 1988, South African Astronomical Observatory Circular, 12, 1

Kilkenny, D., O’Donoghue, D., Koen, C., Stobie, R. S., \& Chen, A. 1997, MNRAS, 287, 867

Kilkenny, D., O’Donoghue, D., Hambly, N., \& MacGillivray, H. 2010, Ap\&SS, 86

Koester, D., Voss, B., Napiwotzki, R., et al. 2009, A\&A, 505, 441

Lamontagne, R., Demers, S., Wesemael, F., Fontaine, G., \& Irwin, M. J. 2000, AJ, 119, 241

Lawrence, A., Warren, S. J., Almaini, O., et al. 2007, MNRAS, 379, 1599

Maxted, P. f. L., Heber, U., Marsh, T. R., \& North, R. C. 2001, MNRAS, 326, 1391

McCook, G. P., \& Sion, E. M. 1999, ApJS, 121, 1

Mengel, J. G., Norris, J., \& Gross, P. G. 1976, ApJ, 204, 488

Morales-Rueda, L., Maxted, P. F. L., Marsh, T. R., North, R. C., \& Heber, U. 2003, MNRAS, 338, 752 
R. Oreiro et al.: A search for new hot subdwarf stars by means of Virtual Observatory tools

Morrissey, P., Conrow, T., Barlow, T. A., et al. 2007, ApJS, 173, 682

Napiwotzki, R., Christlieb, N., Drechsel, H., et al. 2003, The Messenger, 112, 25

Napiwotzki, R., Karl, C. A., Lisker, T., et al. 2004, Ap\&SS, 291, 321

Østensen, R. H. 2006, Balt. Astron., 15, 85

Østensen, R. H., Green, E. M., Bloemen, S., et al. 2010a, MNRAS, 408, L51

Østensen, R. H., Oreiro, R., Solheim, J., et al. 2010b, A\&A, 513, A6

Østensen, R. H., Silvotti, R., Charpinet, S., et al. 2010c, MNRAS, 409, 1470

Østensen, R. H., Silvotti, R., Charpinet, S., et al. 2011, MNRAS, accepted

Randall, S. K., van Grootel, V., Fontaine, G., Charpinet, S., \& Brassard, P. 2009, A\&A, 507, 911

Reed, C. 2005, VizieR Online Data Catalog, 5125, 0

Rhee, J., Seibert, M., Østensen, R. H., et al. 2006, Balt. Astron., 15, 77

Rodríguez-López, C., Ulla, A., \& Garrido, R. 2007, MNRAS, 379, 1123

Rodríguez-López, C., Lynas-Gray, A. E., Kilkenny, D., et al. 2010, MNRAS, 401, 23

Salim, S., \& Gould, A. 2002, ApJ, 575, L83

Sargent, W. L. W., \& Searle, L. 1968, ApJ, 152, 443
Schlegel, D. J., Finkbeiner, D. P., \& Davis, M. 1998, ApJ, 500, 525

Skrutskie, M. F., Cutri, R. M., Stiening, R., et al. 2006, AJ, 131, 1163

Stobie, R. S., Kilkenny, D., O'Donoghue, D., et al. 1997, MNRAS, 287, 848

Stroeer, A., Heber, U., Lisker, T., et al. 2007, A\&A, 462, 269

Thejll, P., Bauer, F., Saffer, R., et al. 1994, ApJ, 433, 819

Ulla, A., \& Thejll, P. 1998, A\&AS, 132, 1

Uytterhoeven, K., Briquet, M., Bruntt, H., et al. 2010, Astron. Nachr., 331, 993

Van Grootel, V., Charpinet, S., Fontaine, G., et al. 2010, ApJ, 718, L97

Vennes, S., Kawka, A., \& Németh, P. 2011, MNRAS, 410, 2095

Wade, R. A., Stark, M. A., Green, R. F., \& Durrell, P. R. 2009, AJ, 138, 606

Wisotzki, L. 1994, in Astronomy from Wide-Field Imaging, ed. H. T. MacGillivray, IAU Symp., 161, 723

Wisotzki, L., Groote, D., Hagen, H., \& Reimers, D. 1991, in Quasar Absorption Lines, ed. P. A. Shaver, E. J. Wampler, \& A. M. Wolfe, 93

Wisotzki, L., Christlieb, N., Bade, N., et al. 2000, A\&A, 358, 77

Woudt, P. A., Kilkenny, D., Zietsman, E., et al. 2006, MNRAS, 371, 1497

Wyder, T. K., Treyer, M. A., Milliard, B., et al. 2005, ApJ, 619, L15 